\title{
CONVERGENCE OF SPECTRA OF GRAPH-LIKE THIN MANIFOLDS
}

\author{
PAVEL EXNER AND OLAF POST
}

\begin{abstract}
We consider a family of compact manifolds which shrinks with respect to an appropriate parameter to a graph. The main result is that the spectrum of the Laplace-Beltrami operator converges to the spectrum of the (differential) Laplacian on the graph with Kirchhoff boundary conditions at the vertices. On the other hand, if the shrinking at the vertex parts of the manifold is sufficiently slower comparing to that of the edge parts, the limiting spectrum corresponds to decoupled edges with Dirichlet boundary conditions at the endpoints. At the borderline between the two regimes we have a third possibility when the limiting spectrum can be described by a nontrivial coupling at the vertices.
\end{abstract}

\section{INTRODUCTION}

Graph models of quantum systems have a long history. Already half a century ago Ruedenberg and Scherr RueS53] used this idea, following a suggestion by L. Pauling, to calculate spectra of aromatic carbohydrate molecules; they achieved a reasonable accuracy for such a simple model. However, a real boom started only from the late eighties when semiconductor graph-type structures became small and clean enough so that coherent effects in the corresponding quantum transport can play the dominating rôle. Due to the rapid progress in fabrication techniques new systems of these type appear every year, making both analysis of graph model properties and their physical justification an urgent task. For the sake of brevity we avoid giving a review of the field with the list of applications and restrict ourselves to quoting the surveys in KoS99, AGHH04, Ku02, $\mathrm{Ku} 04$.

Our aim in the present paper is to contribute to the understanding of ways in which a graph-type description arises from investigation of some really existing systems. To explain what we have in mind, recall that the free Hamiltonian of a graph model is the (differential) Laplacian on the (metric) graph. To define it properly one has to specify the boundary conditions which couple the wave functions at the vertices. They have to define a self-adjoint operator, however, this requirement itself does not specify the conditions uniquely: in a vertex joining $n$ graph edges we have $n^{2}$ free parameters, as observed first in ES89.

Date: November 2, 2018. 
This non-uniqueness represents the main weakness of graph models. A natural idea to mend it is to regard the model in question as a limit case of a more realistic one with a unique Hamiltonian. An appropriate and natural choice is a "thickened graph" composed of thin tubes which have the same topology as the original graph and reduce to it in the limit of a vanishing tube radius. Unfortunately, it is by far not easy to see what happens with spectral and/or scattering properties in such a limit. After a decade-long effort, the spectral convergence in the case when the "thick graph" is planar with Neumann boundary conditions has been solved recently by Kuchment-Zeng [KuZ01, and RubinsteinSchatzman RubS01; Saito [Sa00] showed the convergence of the resolvent. ${ }^{1}$

With the mentioned application to description of quantum wire systems in mind it is clear that an analogous situation in which the tube boundaries are Dirichlet is even more important. Unfortunately, it also very difficult and despite numerous efforts it remains an open problem.

The main insight of the present paper is that these two cases do not exhaust all possible ways in which a family of manifolds can approach a graph. One more choice are manifolds without a boundary of codimension one in $\mathbb{R}^{\nu}, \nu \geq 3$, which encloses the graph like a system of "sleeves" ${ }^{2}$, with the limit consisting of the sleeve diameter shrinking. It is not only a mathematical question; we draw the reader's attention to the fact that such sleeve-shaped tube systems are particularly interesting from the viewpoint of recent efforts to build circuits based on carbon nanotubes. Recall that recently discovered techniques - see, e.g., AMSC01, PRL00, TBG02 — allow to fabricate branched nanotubes and thus in principle objects very similar to the mentioned "sleeved graphs".

On the mathematical side the main contribution of the paper is the treatment of the limit problem in a more abstract setting which covers the "strip graphs" of KuZ01, RubS01 and their generalizations to higher dimensions, as well as the "sleeved graphs" described above. This is achieved by using the internal geometry of such a manifold only, so we need not suppose the latter is embedded in a Euclidean space. Our results even show that the limit is independent of a particular embedding. Only the abstract graph data count.

Our conclusion will be that such a graph limit can give meaning to some type of vertex couplings, in particular, those representing a free motion through the junctions, as well as those which require to extend the graph state space by extra dimensions corresponding to the vertices. To get the full richness of the vertex behaviour, with possible relation to the graph geometry, more general limits will

\footnotetext{
${ }^{1} \mathrm{~A}$ related earlier result can be found in the work of Colin de Verdière CdV86 who used the spectral convergence of thickened graphs to prove that the first non-zero eigenvalue of a compact manifold of dimension greater than 2 can have arbitrary high (finite) multiplicity.

${ }^{2}$ In this context Kuchment and Zeng KuZ01 speak also about sleeves. By this notion they mean graph edges thickened into strips. What we have in mind here is rather a cylindrical surface with the graph edge as its axis.
} 
be needed. To characterize the results as well as the motivation in more details, we need some preliminaries; we will do that in Sections 2.A and 3.E.

Finally we give an application on the spectral convergence result in the case of periodic graphs. In particular, we show the existence of gaps in the spectrum of certain non-compact periodic graph-like manifolds. For example, attaching a loop at each vertex gives rise to spectral gaps (cf. Thm. 9.5).

Let us briefly describe the structure of the paper. In Section 2 we define the Laplacian on a graph and give an abstract eigenvalue comparison tool (Lemma 2.1). In Section 3 we define the graph like manifolds associated to a graph. In Subsection 3.E we motivate the four different limiting procedures on the vertex neighbourhoods discussed in Sections 5 - 8, Our main results are given in Thms. 5.2, 6.2, 17.1 and 8.1. In Section 4 we define the limit procedure of the edge neighbourhoods which remain the same in all cases. The last section (Sec. 9) contains the mentioned applications to periodic graphs.

\section{Preliminaries}

2.A. Laplacian on a graph. Suppose $M_{0}$ is a finite connected graph with vertices $v_{k}, k \in K$ and edges $e_{j}, j \in J$. Suppose furthermore that $e_{j}$ has length $\ell_{j}>0$, i.e., $e_{j} \cong I_{j}:=\left[0, \ell_{j}\right]$. We clearly can make $M_{0}$ into a metric measure space with measure given by $p_{j}(x) d x$ on the edge $e_{j}$ where $p_{j}: I_{j} \longrightarrow(0, \infty)$ is a smooth density function for each $j \in J$. We then have

$$
\begin{gathered}
L_{2}\left(M_{0}\right)=\bigoplus_{j \in J} L_{2}\left(I_{j}, p_{j}(x) d x\right) \\
\|u\|_{M_{0}}^{2}=\sum_{j \in J}\left\|u_{j}\right\|_{I_{j}}^{2}=\sum_{j \in J} \int_{I_{j}}\left|u_{j}(x)\right|^{2} p_{j}(x) d x .
\end{gathered}
$$

We let $\mathcal{H}^{1}\left(M_{0}\right)$ be the completion of

$$
\left\{u \in C\left(M_{0}\right) \mid u_{j}:=u \uparrow_{e_{j}} \in C^{1}\left(I_{j}\right)\right\}
$$

where the closure is taken with respect to the norm

$$
\|u\|_{1, M_{0}}^{2}:=\sum_{j \in J}\left(\left\|u_{j}\right\|_{I_{j}}^{2}+\left\|u_{j}^{\prime}\right\|_{I_{j}}^{2}\right)
$$

Note that the weakly differentiable functions $\mathcal{H}^{1}\left(I_{j}\right)$ on an interval are continuous, therefore $\mathcal{H}^{1}\left(I_{j}\right) \subset C\left(I_{j}\right)$.

Next we associate with the graph a positive quadratic form,

$$
\left\|u^{\prime}\right\|_{M_{0}}^{2}:=\sum_{j \in J}\left\|u_{j}^{\prime}\right\|_{I_{j}}^{2}
$$

for all $u \in \mathcal{H}^{1}\left(M_{0}\right)$. It allows us to define the (differential) Laplacian on the (weighted) graph $M_{0}$ as the unique self-adjoint and non-negative operator $\Delta_{M_{0}}$ associated with the closed form $u \mapsto\left\|u^{\prime}\right\|_{M_{0}}^{2}$ (see [K66, Chapter VI], [RS-1] or 
Da96 for details on quadratic forms). In other words, the operator and the quadratic form are related by

$$
\left\|u^{\prime}\right\|_{M_{0}}^{2}=\left\langle\Delta_{M_{0}} u, u\right\rangle
$$

for $u \in C^{1}\left(M_{0}\right)$ belonging to the domain of $\Delta_{M_{0}}$. On the edge $e_{j}$, the operator $\Delta_{M_{0}}$ is given formally by

$$
\Delta_{M_{0}} u=-\frac{1}{p_{j}(x)}\left(p_{j}(x) u_{j}^{\prime}\right)^{\prime} .
$$

Note that the domain of $\Delta_{M_{0}}$ consists of all functions $u \in C\left(M_{0}\right)$ which are twice weakly differentiable on each edge. Furthermore, each function $u$ satisfies (weighted) Kirchhoff boundary conditions ${ }^{3}$ at each vertex $v_{k}$, i.e.,

$$
\sum_{j, e_{j} \text { meets } v_{k}} p_{j}\left(v_{k}\right) u_{j}^{\prime}\left(v_{k}\right)=0
$$

for all $k \in K$ where the derivative is taken on each edge in the direction away from the vertex. In particular, we assume Neumann boundary conditions at a vertex with only one edge emanating. ${ }^{4}$ If we assume that $p$ is continuous on $M_{0}$, we can omit the factors $p_{j}\left(v_{k}\right)$ in (2.3). Note that different values of $p_{j}\left(v_{k}\right)$ for $j$ can correspond in our limiting result to different radii of the thickened edges which are attached to a vertex neighbourhood (see (4.2) below).

As we have mentioned in the introduction there are other self-adjoint operators which act according to (2.2) on the graph edges but satisfy different boundary conditions at the vertices — see ES89, KoS99] for details. The corresponding quadratic forms differ from (2.1) by an extra term. In general there are many admissible boundary conditions; a graph vertex joining $n$ edges gives rise to a family with $n^{2}$ real parameters. An example is represented by the so-called $\delta$ coupling for which the corresponding domain consists of all functions $u \in C\left(M_{0}\right)$ which are twice weakly differentiable on each edge, and (2.3) is replaced by

$$
\sum_{j, e_{j} \text { meets } v_{k}} p_{j}\left(v_{k}\right) u_{j}^{\prime}\left(v_{k}\right)=\kappa u\left(v_{k}\right)
$$

with a fixed $\kappa \in \mathbb{R}$, where $u\left(v_{k}\right)$ is the common value of all the $u_{j}\left(v_{k}\right)$ at the vertex. One can ask naturally whether such graph Hamiltonians can be obtained from a family of graph-shaped manifolds. In Section 7 we will discuss a particular case of the limiting procedure leading to the spectrum which - although it does not correspond to a graph operator with the generalized boundary condition

\footnotetext{
${ }^{3}$ This is the usual terminology, not quite a fortunate one. The name suggests that the probability current at the vertex obeys the conservation law analogous to Kirchhoff's law in an electric circuit. While this claim is valid, the current conservation requirement is equivalent to selfadjointness and thus also satisfied for the other operators mentioned below.

${ }^{4}$ This hypothesis is made for convenience only and our result will not change if it is replaced by any other boundary condition at the "loose ends", in particular, by Dirichlet or $\theta$-periodic ones (cf. Section 9.A).
} 
described above - is at least similar to that with a $\delta$ coupling. The difference is that in the boundary conditions (2.4) the coupling constant $\kappa$ is replaced by a quantity dependent on the spectral parameter, the corresponding operator being defined not on $L^{2}\left(M_{0}\right)$ but on a slightly enlarged Hilbert space - cf. (7.1)-(7.4).

In Section 6 we obtain another limit operator due to a different limiting procedure. This operator is again no graph operator with boundary conditions as above, but decouples and the graph part corresponds to a fully decoupled operator with Dirichlet boundary conditions at each vertex.

The spectrum of $\Delta_{M_{0}}$ is purely discrete. We denote the corresponding eigenvalues by $\lambda_{k}\left(\Delta_{M_{0}}\right)=\lambda_{k}\left(M_{0}\right), k \in \mathbb{N}$, written in the ascending order and repeated according to multiplicity. With this eigenvalue ordering, we can employ the minmax principle (in the present form it can be found, e.g., in [Da96]): the $k$-th eigenvalue of $\Delta_{M_{0}}$ is expressed as

$$
\lambda_{k}\left(M_{0}\right)=\inf _{L_{k}} \sup _{u \in L_{k} \backslash\{0\}} \frac{\left\|q_{0}(u)\right\|^{2}}{\|u\|^{2}}
$$

where the infimum is taken over all $k$-dimensional subspaces $L_{k}$ of $\mathcal{H}^{1}\left(M_{0}\right)$.

2.B. Comparison of eigenvalues. Let us now formulate a simple consequence of the min-max principle which will be crucial for the proof of our main results. Suppose that $\mathcal{H}, \mathcal{H}^{\prime}$ are two separable Hilbert spaces with the norms $\|\cdot\|$ and $\|\cdot\|^{\prime}$. We need to compare eigenvalues $\lambda_{k}$ and $\lambda_{k}^{\prime}$ of non-negative operators $Q$ and $Q^{\prime}$ with purely discrete spectra defined via quadratic forms $q$ and $q^{\prime}$ on $\mathcal{D} \subset \mathcal{H}$ and $\mathcal{D}^{\prime} \subset \mathcal{H}$. We set $\|u\|_{Q, n}^{2}:=\|u\|^{2}+\left\|Q^{n / 2} u\right\|^{2}$.

Lemma 2.1. Suppose that $\Phi: \mathcal{D} \longrightarrow \mathcal{D}^{\prime}$ is a linear map such that there exist constants $n_{1}, n_{2} \geq 0$ and $\delta_{1}, \delta_{2} \geq 0$ such that

$$
\begin{gathered}
\|u\|^{2} \leq\|\Phi u\|^{\prime 2}+\delta_{1}\|u\|_{Q, n_{1}}^{2} \\
q(u) \geq q^{\prime}(\Phi u)-\delta_{2}\|u\|_{Q, n_{2}}^{2}
\end{gathered}
$$

for all $u \in \mathcal{D}$ and that $\mathcal{D} \subset \operatorname{dom} Q^{\max \left\{n_{1}, n_{2}\right\} / 2}$. Then to each $k$ there is a positive function $\eta_{k}$ given by (2.11) satisfying $\eta_{k}:=\eta\left(\lambda_{k}, \delta_{1}, \delta_{2}\right) \rightarrow 0$ as $\delta_{1}, \delta_{2} \rightarrow 0$, such that

$$
\lambda_{k} \geq \lambda_{k}^{\prime}-\eta_{k}
$$

Proof. Let $\varphi_{1}, \ldots, \varphi_{k}$ be an orthonormal system of eigenvectors corresponding to the eigenvalues $\lambda_{1}, \ldots, \lambda_{k}$. For $u$ in the linear span $E_{k}$ of $\varphi_{1}, \ldots, \varphi_{k}$, we have

$$
\|u\|_{Q, n}^{2} \leq\left(1+\lambda_{k}^{n}\right)\|u\|^{2}
$$


and

$$
\begin{aligned}
\frac{q^{\prime}(\Phi u)}{\|\Phi u\|^{\prime 2}}-\frac{q(u)}{\|u\|^{2}} \leq\left(\frac{q(u)}{\|u\|^{2}} \delta_{1}\|u\|_{Q, n_{1}}^{2}\right. & \left.+\delta_{2}\|u\|_{Q, n_{2}}^{2}\right) \frac{1}{\|\Phi u\|^{\prime 2}} \\
& \leq\left(\lambda_{k}\left(1+\lambda_{k}^{n_{1}}\right) \delta_{1}+\left(1+\lambda_{k}^{n_{2}}\right) \delta_{2}\right) \frac{\|u\|^{2}}{\|\Phi u\|^{\prime 2}}
\end{aligned}
$$

where we have used (2.6) and (2.7) to get the first inequality and (2.8) to get the second one. From relation (2.6) we follow

$$
\left(1-\left(1+\lambda_{k}^{n_{1}}\right) \delta_{1}\right)\|u\|^{2} \leq\|\Phi u\|^{2}
$$

and thus we can estimate the r.h.s. of (2.9) by

$$
\eta_{k}:=\eta\left(\lambda_{k}, \delta_{1}, \delta_{2}\right):=\frac{\lambda_{k}\left(1+\lambda_{k}^{n_{1}}\right) \delta_{1}+\left(1+\lambda_{k}^{n_{2}}\right) \delta_{2}}{1-\left(1+\lambda_{k}^{n_{1}}\right) \delta_{1}}
$$

provided $0 \leq \delta_{1}<1 /\left(1+\lambda_{k}^{n_{1}}\right)$. From (2.10) we also conclude that $\|u\|=0$ holds if $\|\Phi u\|^{\prime}=0$, i.e., that $\Phi\left(E_{k}\right)$ is $k$-dimensional. From the min-max principle applied to the quadratic form $q^{\prime}$ we obtain

$$
\lambda_{k}^{\prime} \leq \sup _{u \in E_{k} \backslash\{0\}} \frac{q^{\prime}(\Phi u)}{\|\Phi u\|^{\prime 2}} \leq \sup _{u \in E_{k} \backslash\{0\}} \frac{q(u)}{\|u\|^{2}}+\eta_{k}=\lambda_{k}+\eta_{k}
$$

which is the desired result.

\section{GRAPH-LIKE MANIFOLDS}

3.A. Laplacian on a manifold. Throughout this paper we study manifolds of dimension $d \geq 2$. For a Riemannian manifold $X$ (compact or not) without boundary we denote by $L_{2}(X)$ the usual $L_{2}$-space of square integrable functions on $X$ with respect to the volume measure $d X$ on $X$. In a chart, the volume measure has the density $(\operatorname{det} G)^{1 / 2}$ with respect to the Lebesgue measure, where $\operatorname{det} G$ is the determinant of the metric tensor $G:=\left(g_{i j}\right)$ in this chart. The norm of $L_{2}(X)$ will be denoted by $\|\cdot\|_{X}$. For $u \in C_{\mathrm{c}}^{\infty}(X)$, the space of compactly supported smooth functions, we set

$$
\check{q}_{X}(u):=\|d u\|_{X}^{2}=\int_{X}|d u|^{2} d X
$$

Here the 1-form $d u$ denotes the exterior derivative of $u$ whose squared norm in coordinates is given by

$$
|d u|^{2}=\sum_{i, j} g^{i j} \partial_{i} u \partial_{j} \bar{u}=G^{-1} \nabla u \cdot \nabla \bar{u}
$$

where $\left(g^{i j}\right)$ is the component representation of the inverse matrix $G^{-1}$.

We denote the closure of the non-negative quadratic form $\check{q}_{X}$ by $q_{X}$. Note that the domain dom $q_{X}$ of the closed quadratic form $q_{X}$ consists of functions in $L_{2}(X)$ such that the weak derivative $d u$ is also square integrable, i.e., $q_{X}(u)<\infty$. 
We define the Laplacian $\Delta_{X}$ (for a manifold without boundary) as the unique self-adjoint and non-negative operator associated with the closed quadratic form $q_{X}$ as in (2.1).

If $X$ is a compact manifold with piecewise smooth boundary $\partial X \neq \emptyset$ we can define the Laplacian with Neumann boundary condition via the closure $q_{X}$ of the quadratic form $\check{q}_{X}$ defined on $C^{\infty}(X)$, the space of smooth functions with derivatives continuous up to the boundary of $X$. Note that the usual conditions on the normal derivative occurs only in the operator domain via the Gauss-Green formula. In a similar way other boundary conditions at $\partial X$ may be introduced. The spectrum of $\Delta_{X}$ (with any boundary condition if $\partial X \neq \emptyset$ ) is purely discrete as long as $X$ is compact and the boundary conditions are local. We denote the corresponding eigenvalues by $\lambda_{k}\left(\Delta_{X}\right)=\lambda_{k}(X), k \in \mathbb{N}$, written in increasing order and repeated according to multiplicity.

3.B. General estimates on manifolds. We will employ (partial) averaging processes on edge and vertex neighbourhoods which correspond to projection onto the lowest (transverse) mode. We start with such a general Poincaré-type estimate:

Lemma 3.1. Let $X$ be a connected, compact manifold with smooth boundary $\partial X$. For $u \in \mathcal{H}^{1}(X)$ define the constant function $u_{0}(x):=\frac{1}{\operatorname{vol} X} \int_{X} u d X$. Then we have $\left\|u_{0}\right\|_{X}^{2} \leq\|u\|_{X}^{2}$,

$$
\left\|u-u_{0}\right\|_{X}^{2} \leq \frac{1}{\lambda_{2}^{\mathrm{N}}(X)}\|d u\|_{X}^{2} \quad \text { and } \quad\|u\|_{X}^{2}-\left\|u_{0}\right\|_{X}^{2} \leq \frac{1}{\delta \lambda_{2}^{\mathrm{N}}(X)}+\delta\|u\|_{X}^{2}
$$

for $\delta>0$.

Proof. The first inequality follows directly from Cauchy-Schwarz. For the second one, note that $u-u_{0}$ is orthogonal to the first eigenfunction of the Neumann Laplacian. By the min-max principle we obtain

$$
\lambda_{2}^{\mathrm{N}}(X)\left\|u-u_{0}\right\|_{X}^{2} \leq\left\|d\left(u-u_{0}\right)\right\|_{X}^{2}=\|d u\|_{X}^{2} .
$$

Since $X$ is connected, we have $\lambda_{2}^{\mathrm{N}}(X)>0$. The last inequality follows from

$$
\left|\|u\|^{2}-\left\|u_{0}\right\|^{2}\right| \leq 2\left\|u-u_{0}\right\|\|u\| \leq \frac{1}{\delta}\left\|u-u_{0}\right\|^{2}+\delta\|u\|^{2}
$$

for all $\delta>0$.

Next, we need the following continuity of the map which restricts a function on $X$ to the boundary $\partial X$. To this aim we use standard Sobolev embedding theorems:

Lemma 3.2. There exists a constant $c_{1}>0$ depending only on $X$ and the metric $g$ such that

for all $u \in \mathcal{H}^{1}(U)$.

$$
\left\|u \uparrow_{\partial X}\right\|_{\partial X}^{2} \leq c_{1}\left(\|u\|_{X}^{2}+\|d u\|_{X}^{2}\right)
$$


Proof. See e.g. TTay96, Ch. 4, Prop. 4.5]. An alternative proof similar to the proof of Lemma 6.7 exists, and follows easily from (6.20) together with a cut-off function.

3.C. Definition of the graph-like manifold. For each $0<\varepsilon \leq 1$ we associate with the graph $M_{0}$ a compact and connected Riemannian manifold $M_{\varepsilon}$ of dimen-
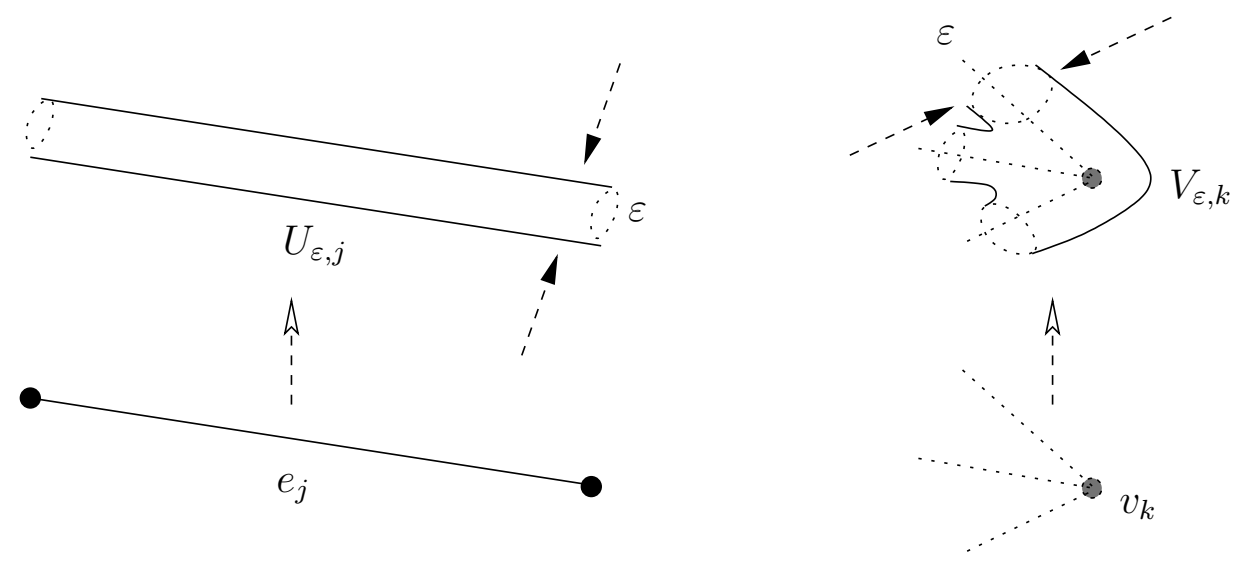

Figure 1. The associated edge and vertex neighbourhoods with $F=\mathbb{S}^{1}$, i.e., $U_{\varepsilon, j}$ and $V_{\varepsilon, k}$ are 2-dimensional manifolds with boundary.

sion $d \geq 2$ equipped with a metric $g_{\varepsilon}$ to be specified below. We suppose that $M_{\varepsilon}$ is the union of compact subsets $U_{\varepsilon, j}$ and $V_{\varepsilon, k}$ such that the interiors of $U_{\varepsilon, j}$ and $V_{\varepsilon, k}$ are mutually disjoint for all possible combinations of $j \in J$ and $k \in K$. We think of $U_{\varepsilon, j}$ as the thickened edge $e_{j}$ and of $V_{\varepsilon, k}$ as the thickened vertex $v_{k}$ (see Figures 1 and 21). Note that Figure 2 describes the situation only roughly, since it assumes that $M_{\varepsilon}$ is embedded in $\mathbb{R}^{\nu}$. More correctly, we should think of $M_{\varepsilon}$ as an abstract manifold obtained by identifying the appropriate boundary parts of $U_{\varepsilon, j}$ and $V_{\varepsilon, k}$ via the connection rules of the graph $M_{0}$. This manifold need not to be embedded, but the situation when $M_{\varepsilon}$ is a submanifold of $\mathbb{R}^{\nu}(\nu \geq d)$ can be viewed also in this abstract context (see Example 4.1).

As a matter of convenience we assume that $U_{\varepsilon, j}$ and $V_{\varepsilon, k}$ are independent of $\varepsilon$ as manifolds, i.e., only their metric $g_{\varepsilon}$ depend on $\varepsilon$. This can be achieved in the following way: for the edge regions we assume that $U_{\varepsilon, j}$ is diffeomorphic to $I_{j} \times F$ for all $0<\varepsilon \leq 1$ where $F$ denotes a compact and connected manifold (with or without a boundary) of dimension $m:=d-1$. For the vertex regions we assume that the manifold $V_{\varepsilon, k}$ is diffeomorphic to an $\varepsilon$-independent manifold $V_{k}$ for $0<\varepsilon \leq 1$. Pulling back the metrics to the diffeomorphic manifold we may assume that the underlying differentiable manifold is independent of $\varepsilon$. Therefore, $U_{\varepsilon, j}=U_{j}=I_{j} \times F$ and $V_{\varepsilon, k}=V_{k}$ with an $\varepsilon$-depending metric $g_{\varepsilon}$. 

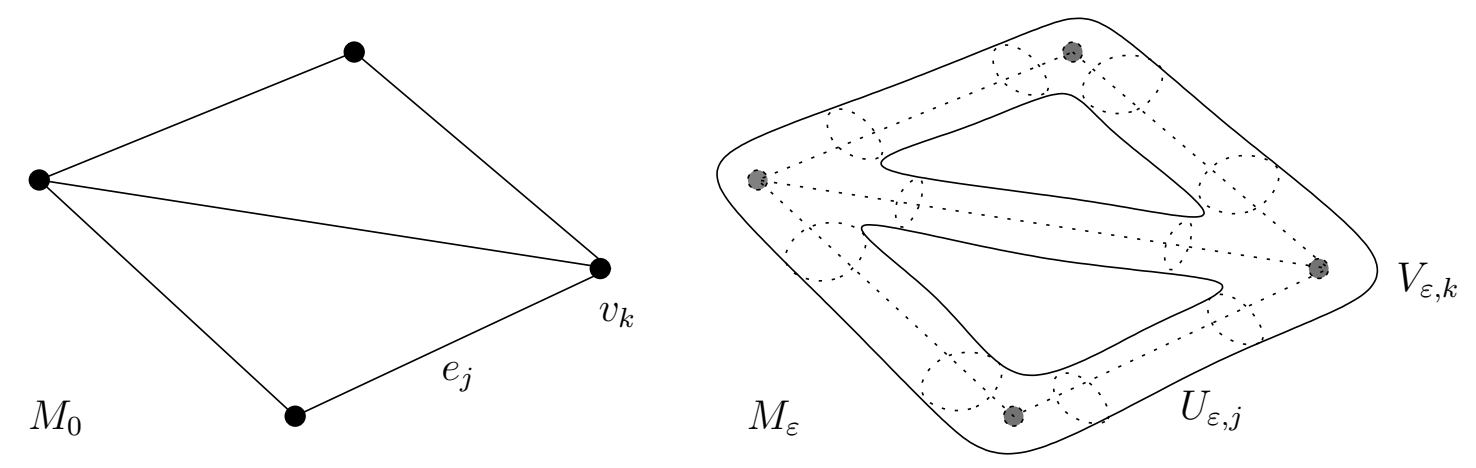

Figure 2. On the left, we have the graph $M_{0}$, on the right, the associated graph-like manifold (in this case, $F=\mathbb{S}^{1}$ and $M_{\varepsilon}$ is a 2-dimensional manifold).

For further purposes, we need a decomposition of $e_{j} \cong I_{j}$ into two halves. We reverse the orientation of one such half so that each half is directed away from its adjacent vertex and collect all halves $I_{j k}$ ending at the vertex $v_{k}$, i.e., $j \in J_{k}$, where

$$
J_{k}:=\left\{j \in J \mid e_{j} \text { meets } v_{k}\right\}^{5}
$$

We denote $U_{j k}:=I_{j k} \times F$ (and similar notation with subscript $\varepsilon$ ).

For further references, we denote the midpoint of the edge $e_{j} \cong I_{j}$ by $x_{j}^{*}$ and the endpoint of $I_{j}$ corresponding to the edge $v_{k}$ by $x_{j k}^{0}$, e.g., $I_{j k}=\left[x_{j}^{*}, x_{j k}^{0}\right]$.

3.D. Notation. In the sequel, we are going to suppress the edge and vertex subscripts $j$ and $k$ unless a misunderstanding may occur. Similarly we set, e.g., $U:=U_{1}$, in other words we omit the subscript $\varepsilon$ if we only mean the underlying $\varepsilon$-independent manifold with metric $g_{1}$, i.e., if we fix $\varepsilon=1$.

3.E. Motivation for the different limit operators. Let us briefly motivate why the limit operator of $\Delta_{M_{\varepsilon}}$ as $\varepsilon \rightarrow 0$ should depend on the volume decay of the vertex neighbourhoods $V_{\varepsilon, k}$ in comparison with $\operatorname{vol}_{d-1} \partial V_{\varepsilon, k}\left(\operatorname{or}_{\operatorname{vol}_{d}} U_{\varepsilon, j}\right.$, which is of the same order when $\varepsilon \rightarrow 0$ as we will see in Section 4). For simplicity, we assume that the radius of the transversal direction on the edge $U_{\varepsilon, j}$ is $\varepsilon$ (i.e., $\left.p_{j} \equiv 1\right)$. The assumptions on the edge neighbourhoods will be specified in the next section. We stress that our aim in this subsection is to present a heuristic idea, not a proof (for a suitable reasoning cf. RueS53] or [Ku02]).

Suppose $\varphi=\varphi_{\varepsilon}$ is an eigenfunction of $\Delta_{M_{\varepsilon}}$ w.r.t the eigenvalue $\lambda=\lambda_{\varepsilon}$. By the Gauss-Green formula, we have at the vertex $V_{\varepsilon}=V_{\varepsilon, k}$

$$
\lambda \int_{V_{\varepsilon}} \varphi \bar{u} d V_{\varepsilon}=\int_{V_{\varepsilon}}\langle d \varphi, d u\rangle d V_{\varepsilon}+\int_{\partial V_{\varepsilon}} \partial_{\mathrm{n}} \varphi \bar{u} d \partial V_{\varepsilon}
$$

\footnotetext{
${ }^{5}$ For each loop $e_{j}$ at $v_{k}$, i.e., each edge beginning and ending at $v_{k}$, we need to replace the label $j$ by two distinct labels $j_{1}, j_{2}$ belonging to $J_{k}$ in order to collect both halfs of the edge.
} 
for all $u \in \mathcal{H}^{1}\left(M_{\varepsilon}\right)$. Assume that $\lambda_{\varepsilon} \rightarrow \lambda_{0}$ and $\varphi_{\varepsilon} \rightarrow \varphi_{0}=\left(\varphi_{0, j}\right)_{j}$.

If the vertex volume $\operatorname{vol}_{d} V_{\varepsilon}$ decays faster than the boundary area $\operatorname{vol}_{d-1} \partial V_{\varepsilon}$ only the boundary integral over $\partial V_{\varepsilon}$ survives in the limit $\varepsilon \rightarrow 0$ and leads to

$$
0=\sum_{j \in J_{k}} \varphi_{0, j}^{\prime}\left(v_{k}\right)
$$

which is exactly the Kirchhoff boundary condition mentioned above in (2.3). This fast decaying vertex volume case will be treated in Section [5.

If the vertex volume decays slower than $\operatorname{vol}_{d-1} \partial V_{\varepsilon}$, the integrals over $V_{\varepsilon}$ are dominant. In this case, $\operatorname{vol} V_{\varepsilon, k} \gg \operatorname{vol} U_{\varepsilon, j}$ and only slowly varying eigenfunctions on $V_{\varepsilon, k}$ lead to bounded eigenvalues $\lambda=\lambda_{\varepsilon}$. Since $\operatorname{vol} V_{\varepsilon, k} \gg \operatorname{vol} U_{\varepsilon, j}$, normalized eigenfunctions are nearly vanishing on $V_{\varepsilon, k}$ viewed from the scale on $U_{\varepsilon, j}$. This roughly explains, why we end up with a decoupled operator with Dirichlet boundary conditions on $M_{0}$ plus extra zero eigenmodes at the vertices (the zero eigenmodes also survive the limit $\varepsilon \rightarrow 0$ ). This slowly decaying vertex volume case will be discussed in Section [6]

In the borderline case when $\operatorname{vol}_{d} V_{\varepsilon} \approx \operatorname{vol}_{d-1} \partial V_{\varepsilon}$, we also expect the eigenfunctions to vary slowly on $V_{\varepsilon, k}$ ( since $\operatorname{vol}_{d} V_{\varepsilon, k} \rightarrow 0$ ), so the integral over $\langle d \varphi, d u\rangle$ should tend to 0 , and in the limit

$$
\lambda_{0} \varphi_{0}\left(v_{k}\right)=\sum_{j \in J_{k}} \varphi_{0, j}^{\prime}\left(v_{k}\right) .
$$

This borderline case will be treated in Section 7

If vol $V_{\varepsilon, k}$ does not tend to 0 , i.e., when $V_{\varepsilon, k}$ tends to a compact $d$-dimensional manifold $V_{0, k}$ without boundary (and not to a point as in the cases above), we still expect a decoupled operator with Dirichlet boundary conditions on the edges by the same arguments as in the slowly decaying case. In addition, not only the lowest eigenmode of $V_{\varepsilon, k}$ but all eigenmodes survive, i.e., the limit operator should consist of the direct sum of all Dirichlet Laplacians on the edges plus the Laplacians on $V_{0, k}, k \in K$. This non-decaying vertex volume case will be treated in Section 8

It requires an extra effort to prove rigorously the conclusions of the above reasoning; recall that we have assumed e.g. that $\lambda_{\varepsilon} \rightarrow \lambda_{0}$ (which we want to show in this paper) and $\left\|\varphi_{\varepsilon}\right\|_{\infty},\left\|d \varphi_{\varepsilon}\right\|_{\infty} \leq c$ which is in general not true for normalized $\left(L_{2^{-}}\right)$eigenfunctions since vol $M_{\varepsilon} \rightarrow 0$ as $\varepsilon \rightarrow 0$.

\section{EdGe NeIghBourhoods}

4.A. Definition of the thickened edges. Suppose that $U=I \times F$ with metric $g_{\varepsilon}$, where $I$ corresponds to some (part of an) edge and $F$ denotes (as before) a compact and connected Riemannian manifold of dimension $m=d-1$ with metric $h$ with or without boundary (we always assume that the corresponding Laplacian on $M_{\varepsilon}$ satisfies Neumann boundary conditions on the boundary part coming from 
$\partial F)$. For simplicity we assume that vol $F=1$. We define another metric $\tilde{g}_{\varepsilon}$ on $U_{\varepsilon}$ by

$$
\tilde{g}_{\varepsilon}:=d x^{2}+\varepsilon^{2} r_{j}^{2}(x) h(y), \quad(x, y) \in U_{j}=I_{j} \times F
$$

where

$$
r_{j}(x):=\left(p_{j}(x)\right)^{1 / m}
$$

defines a smooth function (specifying the radius of the fibre $\{x\} \times F$ at the point $x$ ), where $p_{j}$ is the density function on the edge $e_{j}$ introduced in Section 2.

We denote by $G_{\varepsilon}$ and $\tilde{G}_{\varepsilon}$ the $d \times d$-matrices associated to the metrics $g_{\varepsilon}$ and $\tilde{g}_{\varepsilon}$ with respect to the coordinates $(x, y)$ (here $y$ stands for suitable coordinates on $F$ ) and assume that the two metrics coincide up to an error term as $\varepsilon \rightarrow 0$, more specifically

$$
G_{\varepsilon}=\tilde{G}_{\varepsilon}+\left(\begin{array}{cc}
o(1) & o(\varepsilon) \\
o(\varepsilon) & o\left(\varepsilon^{2}\right)
\end{array}\right)=\left(\begin{array}{cc}
1+o(1) & o(\varepsilon) \\
o(\varepsilon) & \varepsilon^{2} r_{j} H+o\left(\varepsilon^{2}\right)
\end{array}\right)
$$

i.e.,

$$
g_{\varepsilon, x x}=1+o(1), \quad g_{\varepsilon, x y_{\alpha}}=o(\varepsilon), \quad g_{\varepsilon, y_{\alpha} y_{\beta}}=\varepsilon^{2} r_{j}^{2}(x) h_{\alpha \beta}(y)+o\left(\varepsilon^{2}\right) .
$$

uniformly on $U$. To summarize, we assume that the metric $g_{\varepsilon}$ is equal to the product metric $\tilde{g}_{\varepsilon}$ up to error terms.

This is a central assumption in our construction which describes how in fact the family of manifolds shrinks to the graph $M_{0}$. One of the reasons why we introduce a pair of metrics will become clear in the following two examples. While our construction uses intrinsic metric properties of the manifolds only, we want it to be applicable to manifolds embedded into some Euclidean space $\mathbb{R}^{\nu}$. It will be one of our aims to show that within the prescribed error margin such a "practically important" metric yields the same result as the product metric which is easier to handle.

In particular, our results show that the convergence is independent of the chosen embedding.

Example 4.1. Embedded graphs. Note that it is impossible to embed our graph neighbourhood $M_{\varepsilon}$ if the cylindrical sleeves have the same length as the underlying graph edges, but it can be achieved with the length shortened by a factor of order $o(1)$. In this sense, we recover the situation treated in KuZ01, i.e., $M_{0}$ embedded in $\mathbb{R}^{2}$ and $F=[-1,1]$ and $M_{\varepsilon}$ being a suitable $\varepsilon$-neighbourhood of $M_{0}$.

In the same way, we can treat the graph $M_{0}$ embedded in $\mathbb{R}^{3}$ with $M_{\varepsilon}$ being the surface of some pipeline network (i.e. $F=\mathbb{S}^{1}$ ).

Example 4.2. Curved edges and variable transversal radius. Suppose $U_{\varepsilon}$ is the $\varepsilon$-neighbourhood of a smooth curve $\vec{\gamma}=\vec{\gamma}_{j}: I_{j} \longrightarrow \mathbb{R}^{d}$ parameterized by arc-length. If, e.g., $\nu=d=2$ and $F=[-1,1]$ a chart is given by

$$
\Psi: I_{j} \times[-1,1] \longrightarrow U_{\varepsilon, j}, \quad(x, y) \mapsto \vec{\gamma}(x)+\varepsilon r_{j}(x) y \vec{n}(x),
$$


i.e., we thicken the curve $\vec{\gamma}$ in its normal direction $\vec{n}(x)$ at the point $\vec{\gamma}(x)$ by the factor $\varepsilon r(x)=\varepsilon r_{j}(x)$. The corresponding metric in $(x, y)$-coordinates is given by

$$
G_{\varepsilon}=\left(\begin{array}{cc}
(1+\varepsilon \kappa y r)^{2}+\varepsilon^{2} y^{2} \dot{r}^{2} & \varepsilon^{2} r \dot{r} y \\
\varepsilon^{2} r \dot{r} y & \varepsilon^{2} r^{2}
\end{array}\right)=\left(\begin{array}{cc}
1+O(\varepsilon) & O\left(\varepsilon^{2}\right) \\
O\left(\varepsilon^{2}\right) & \varepsilon^{2} r^{2}
\end{array}\right)
$$

where $\kappa:=\dot{\gamma}_{1} \ddot{\gamma}_{2}-\dot{\gamma}_{2} \ddot{\gamma}_{2}$ is the curvature of the generating curve $\vec{\gamma}$. Therefore, the error term $o(1)$ comes from the curvature of the embedded curve $\vec{\gamma}$ whereas the off-diagonal error terms come from the variable radius of the transversal direction (note that $\dot{r}=0$ if $r(x)$ is constant). Curvature induced effects in the thin tube limit are well understood - see, e.g., DE95.

4.B. Estimates on the thickened edges. Following the philosophy explained in the previous subsection, we start with pointwise estimates where we compare the product metric $\tilde{g}_{\varepsilon}$ with the original metric $g_{\varepsilon}$. Note that the assumption (4.3), while fully sufficient for our purposes, is optimal in a sense, i.e., that the following lemma ceases to be valid if we weaken its hypothesis even slightly.

Lemma 4.3. Suppose that $g_{\varepsilon}, \tilde{g}_{\varepsilon}$ are given as in (4.1) and (4.3), then

$$
\begin{aligned}
\left(\operatorname{det} G_{\varepsilon}\right)^{\frac{1}{2}} & =(1+o(1))\left(\operatorname{det} \tilde{G}_{\varepsilon}\right)^{\frac{1}{2}} \\
g_{\varepsilon}^{x x} & :=\left(G_{\varepsilon}^{-1}\right)_{x x}=1+o(1) \\
\left|d_{x} u\right|^{2} & \leq(1+o(1))|d u|_{g_{\varepsilon}}^{2} \\
\left|d_{F} u\right|_{h}^{2} & \leq o(\varepsilon)|d u|_{g_{\varepsilon}}^{2}
\end{aligned}
$$

where $d_{x}$ and $d_{F}$ are the (exterior) derivative with respect to $x \in I$ and $y \in F$, respectively. All the estimates are uniform in $(x, y)$ as $\varepsilon \rightarrow 0$.

Proof. The first equation follows from

$$
\begin{aligned}
\operatorname{det}\left(G_{\varepsilon} \tilde{G}_{\varepsilon}^{-1}\right) & =\operatorname{det}\left(\begin{array}{cc}
1+o(1) & o(\varepsilon) \\
o(\varepsilon) & \varepsilon^{2} H+o\left(\varepsilon^{2}\right)
\end{array}\right)\left(\begin{array}{cc}
1 & 0 \\
0 & \varepsilon^{-2} H^{-1}
\end{array}\right) \\
& =\operatorname{det}\left(\begin{array}{cc}
1+o(1) & o\left(\varepsilon^{-1}\right) \\
o(\varepsilon) & \mathbb{1}+o(1)
\end{array}\right)=1+o(1) .
\end{aligned}
$$

For the second one, we consider the upper left component of

$$
\begin{aligned}
G_{\varepsilon}^{-1} & -\tilde{G}_{\varepsilon}^{-1}=-\tilde{G}_{\varepsilon}^{-1}\left(G_{\varepsilon}-\tilde{G}_{\varepsilon}\right) \tilde{G}_{\varepsilon}^{-1}+o\left(G_{\varepsilon}-\tilde{G}_{\varepsilon}\right) \\
\quad= & \left(\begin{array}{cc}
1 & 0 \\
0 & O\left(\varepsilon^{-2}\right)
\end{array}\right)\left(\begin{array}{cc}
o(1) & o(\varepsilon) \\
o(\varepsilon) & o\left(\varepsilon^{2}\right)
\end{array}\right)\left(\begin{array}{cc}
1 & 0 \\
0 & O\left(\varepsilon^{-2}\right)
\end{array}\right)+o(1)=\left(\begin{array}{cc}
o(1) & o\left(\varepsilon^{-1}\right) \\
o\left(\varepsilon^{-1}\right) & o\left(\varepsilon^{-2}\right)
\end{array}\right) .
\end{aligned}
$$

Inequality (4.6) is equivalent to

$$
\left(\begin{array}{ll}
1 & 0 \\
0 & 0
\end{array}\right) \leq(1+o(1)) G_{\varepsilon}^{-1}
$$


in the sense of quadratic forms. This will be true if we show that

$$
\left(\begin{array}{cc}
1 & 0 \\
0 & \delta \mathbb{1}
\end{array}\right) \leq(1+o(1)) G_{\varepsilon}^{-1}
$$

for some $\delta>0$, where $\mathbb{1}$ is the $m \times m$ unit matrix, which in turn means

$$
(1+o(1))\left(\begin{array}{cc}
1 & 0 \\
0 & \delta^{-1} \mathbb{1}
\end{array}\right) \geq G_{\varepsilon}
$$

However,

$$
G_{\varepsilon}=\tilde{G}_{\varepsilon}+\left(\begin{array}{cc}
o(1) & o(\varepsilon) \\
o(\varepsilon) & o\left(\varepsilon^{2}\right)
\end{array}\right)=\left(\begin{array}{cc}
1+o(1) & 0 \\
0 & O\left(\varepsilon^{2}\right)
\end{array}\right)+\left(\begin{array}{cc}
0 & o(\varepsilon) \\
o(\varepsilon) & 0
\end{array}\right)
$$

and the eigenvalues of the last matrix are of order $o(\varepsilon)$, so

$$
G_{\varepsilon} \leq\left(\begin{array}{cc}
1+o(1) & 0 \\
0 & O\left(\varepsilon^{2}\right)
\end{array}\right)+o(\varepsilon) \mathbb{1}=\left(\begin{array}{cc}
1+o(1) & 0 \\
0 & o(\varepsilon)
\end{array}\right) \leq(1+o(1))\left(\begin{array}{cc}
1 & 0 \\
0 & c \mathbb{1}
\end{array}\right)
$$

for some constant $c>0$, and therefore it is sufficient to choose $\delta<c^{-1}$. The proof of inequality (4.7) is similar.

4.C. Notation. The tilde in a symbol refers always to the product metric $\tilde{g}_{\varepsilon}$. We denote, e.g., by $\tilde{U}_{\varepsilon}$ the manifold $U_{\varepsilon}$ with metric $\tilde{g}_{\varepsilon}$ and (abusing the notation a little bit) employ the symbol $U_{\varepsilon}$ for the manifold $U_{\varepsilon}$ with the metric $g_{\varepsilon}$.

As a motivation for the above choice of the metrics, let us calculate the norm of $u \in L_{2}\left(\tilde{U}_{\varepsilon}\right)$ for a function $u$ which is independent of the second argument $y \in F$, i.e. $u(x, y)=u(x)$. This yields

$$
\begin{aligned}
& \|u\|_{\tilde{U}_{\varepsilon}}^{2}=\int_{U_{\varepsilon}}|u|^{2} d \tilde{U}_{\varepsilon}=\int_{I} \int_{F}|u(x)|^{2}\left(\operatorname{det} \tilde{G}_{\varepsilon}\right)^{\frac{1}{2}}(x, y) d x d y \\
& =\varepsilon^{m} \int_{I}|u(x)|^{2} r^{m}(x) d x \int_{F}(\operatorname{det} H)^{\frac{1}{2}}(y) d y=\varepsilon^{m}\|u\|_{I}^{2} \operatorname{vol}(F)=\varepsilon^{m}\|u\|_{I}^{2} .
\end{aligned}
$$

4.D. Transversal averaging. We will employ averaging processes on edge neighbourhoods $U_{\varepsilon}=U_{\varepsilon, j}$ which correspond to projection onto the lowest transverse mode:

$$
N u(x)=N_{j} u(x):=\int_{F} u(x, \cdot) d F
$$

Note that $N u(x)$ is well defined for $u \in \mathcal{H}^{1}\left(U_{\varepsilon}\right)$, and moreover,

$$
\varepsilon^{m}\|N u\|_{I}^{2}=\|N u\|_{\tilde{U}_{\varepsilon}}^{2} \leq\|u\|_{\tilde{U}_{\varepsilon}}^{2}=(1+o(1))\|u\|_{U_{\varepsilon}}^{2}
$$

in view of eqs. (4.8), (4.4), and the Cauchy-Schwarz inequality.

In the following two lemmas we compare a function $u$ and its derivative $d u$ with the normal averages $N u$ and $d_{x} N u$, respectively. Note that for the next lemma, 4.10) is not enough; we also need the reverse inequality: 
Lemma 4.4. For any $u \in \mathcal{H}^{1}\left(U_{\varepsilon}\right)$ we have

$$
\|u\|_{U_{\varepsilon}}^{2}-\left\|\varepsilon^{m / 2} N u\right\|_{I}^{2} \leq o\left(\varepsilon^{1 / 2}\right)\left(\|u\|_{U_{\varepsilon}}^{2}+\|d u\|_{U_{\varepsilon}}^{2}\right) .
$$

Proof. Applying Lemma 3.1] with $X=F$ we get

$$
\|u(x, \cdot)\|_{F}^{2}-|N u(x)|^{2} \leq \frac{1}{\delta \lambda_{2}^{\mathrm{N}}(F)}\left\|d_{F} u(x, \cdot)\right\|_{F}^{2}+\delta\|u(x, \cdot)\|_{F}^{2} .
$$

Next we integrate over $I_{j}$ and obtain

$$
\|u\|_{\tilde{U}_{\varepsilon}}^{2}-\varepsilon^{m}\|N u\|_{I_{j}}^{2} \leq \frac{o(\varepsilon)}{\delta \lambda_{2}^{\mathrm{N}}(F)} \int_{\tilde{U}_{\varepsilon, j}}|d u|_{g_{\varepsilon}}^{2} d \tilde{U}_{\varepsilon, j}+\delta\|u\|_{\tilde{U}_{\varepsilon, j}}^{2}
$$

using estimate (4.7). We put $\delta:=\sqrt{o(\varepsilon)}$ and apply (4.4) to obtain the result for the manifold $U_{\varepsilon, j}$.

Lemma 4.5. For any $u \in \mathcal{H}^{1}\left(U_{\varepsilon}\right)$ we have

$$
\left\|\varepsilon^{m / 2}(N u)^{\prime}\right\|_{I}^{2}-\|d u\|_{U_{\varepsilon}}^{2} \leq o(1)\|d u\|_{U_{\varepsilon}}^{2} .
$$

Proof.

$$
\begin{aligned}
& \left\|\varepsilon^{m / 2}(N u)^{\prime}\right\|_{I}^{2}=\left\|\varepsilon^{m / 2} N\left(d_{x} u\right)\right\|_{I}^{2} \leq(1+o(1))\left\|d_{x} u\right\|_{U_{\varepsilon}}^{2} \\
& \quad=(1+o(1)) \int_{U_{\varepsilon}}\left|d_{x} u\right|^{2} d U_{\varepsilon} \leq(1+o(1)) \int_{U_{\varepsilon}}|d u|_{g_{\varepsilon}}^{2} d U_{\varepsilon}=(1+o(1))\|d u\|_{U_{\varepsilon}}^{2}
\end{aligned}
$$

holds in view of estimate (4.10) and (4.6).

Next we need a pointwise estimate on the behaviour of $N u$ at the boundary.

Lemma 4.6. We have

$$
\left|N u\left(x^{0}\right)\right|^{2} \leq O\left(\varepsilon^{-m}\right)\left(\|u\|_{U_{\varepsilon}}^{2}+\|d u\|_{U_{\varepsilon}}^{2}\right)
$$

for all $u \in \mathcal{H}^{1}\left(U_{\varepsilon}\right)$, where $x^{0} \in \partial I$.

Proof. One estimates

$\left|N u\left(x^{0}\right)\right|^{2} \leq \int_{F}\left|u\left(x^{0}, y\right)\right|^{2} d F(y) \leq c_{1}\left(\|u\|_{U}^{2}+\left\|d_{x} u\right\|_{U}^{2}\right) \leq O\left(\varepsilon^{-m}\right)\left(\|u\|_{U_{\varepsilon}}^{2}+\|d u\|_{U_{\varepsilon}}^{2}\right)$ by Lemma 3.2 with $X=U$, (4.6) and (4.4).

\section{Fast decaying Vertex volume}

5.A. Definition of the thickened vertices. Remember that $V_{\varepsilon, k}=V_{k}$ as manifold, whereas $g_{\varepsilon}$ denotes the $\varepsilon$-depending metric on $V_{\varepsilon, k}$. Let $g:=g_{1}$, then we assume that

$$
c_{-} \varepsilon^{2} g \leq g_{\varepsilon} \leq c_{+} \varepsilon^{2 \alpha} g
$$

in the sense that there are constants $c_{-}, c_{+}>0$ such that

$$
c_{-} \varepsilon^{2} g(x)(v, v) \leq g_{\varepsilon}(x)(v, v) \leq c_{+} \varepsilon^{2 \alpha} g(x)(v, v)
$$


for all $v \in T_{x} V_{k}$ and all $x \in V_{k}$. The number $\alpha$ in the exponent is assumed to satisfy the inequalities

$$
\frac{d-1}{d}<\alpha \leq 1
$$

notice that $\alpha \leq 1$ is needed for (5.1) to make sense with $0<\varepsilon \leq 1$. Thus the edge and vertex parts of the manifold need not shrink at the same rate but the vertex shrinking should not be too slow than that of the edges. This hypothesis expressed by (5.1) plays a central rôle here; other shrinking regimes will be discussed in the following sections.

Note that the manifold $V_{\varepsilon, k}$ shrinks at most as $\varepsilon$ (in each direction) by the lower bound in (5.1). This ensures that a global smooth metric $g_{\varepsilon}$ exists on $M_{\varepsilon}$ with the requirements on $U_{\varepsilon, j}$ and $V_{\varepsilon, k}$. Therefore, we do not need an intermediate part (called bottle neck) between the edge and vertex neighbourhoods interpolating between the different scalings as in Sections [6 and 7.

We easily obtain the following global estimate from (5.1):

Lemma 5.1. There are $c_{1}^{ \pm}, c_{2}^{ \pm}>0$ such that

$$
\begin{gathered}
c_{1}^{-} \varepsilon^{d}\|u\|_{V}^{2} \leq\|u\|_{V_{\varepsilon}}^{2} \leq c_{1}^{+} \varepsilon^{\alpha d}\|u\|_{V}^{2} \\
c_{2}^{-} \varepsilon^{d-2 \alpha}\|d u\|_{V}^{2} \leq\|d u\|_{V_{\varepsilon}}^{2} \leq c_{2}^{+} \varepsilon^{\alpha d-2}\|d u\|_{V}^{2}
\end{gathered}
$$

for all $u \in \mathcal{H}^{1}\left(V_{\varepsilon}\right)=\mathcal{H}^{1}(V)$.

5.B. Convergence of the spectra. The limit operator will concentrate only on the edge part in this case, therefore we define

$$
\mathcal{H}_{0}:=L_{2}\left(M_{0}\right), \quad \mathcal{D}_{0}:=\mathcal{H}^{1}\left(M_{0}\right), \quad q_{0}(u):=\left\|u^{\prime}\right\|_{M_{0}}^{2}=\sum_{j}\left\|u_{j}^{\prime}\right\|_{I_{j}}^{2},
$$

i.e., the limit operator $Q_{0}$ is $\Delta_{M_{0}}$ (see Def. (2.2)). If the transversal manifold $F$ has boundary, we assume that $\Delta_{M_{\varepsilon}}$ satisfies Neumann boundary conditions. With the above preliminaries we can finally formulate the main result of this section:

Theorem 5.2. Under the stated assumptions $\lambda_{k}\left(M_{\varepsilon}\right) \rightarrow \lambda_{k}\left(M_{0}\right)$ as $\varepsilon \rightarrow 0$.

Recall that the eigenvalues $\lambda_{k}\left(M_{\varepsilon}\right)$ are by assumption ordered in the ascending order, multiplicity taken into account, so the label of a particular eigenvalue curve may change as $\varepsilon$ moves. The spectrum of the Laplacian on $M_{\varepsilon}$ is in general richer than that of the graph and a part of the eigenvalues escapes to $+\infty$ as $\varepsilon \rightarrow 0$; the proof presented below shows that this happens, roughly speaking, for all states with the transverse part of the eigenfunction orthogonal to the ground state.

Our aim is to find a two sided estimate on each eigenvalue $\lambda_{k}\left(M_{\varepsilon}\right)$ by means of $\lambda_{k}\left(M_{0}\right)$ with an error which is $o(1)$ w.r.t. the parameter $\varepsilon$. 
5.C. An upper bound. The mentioned upper eigenvalue estimate now reads as follows:

Theorem 5.3. $\lambda_{k}\left(M_{\varepsilon}\right) \leq \lambda_{k}\left(M_{0}\right)+o(1)$ holds as $\varepsilon \rightarrow 0$.

To prove it, we define the transition operator by

$$
\Phi_{\varepsilon} u(z):= \begin{cases}\varepsilon^{-m / 2} u\left(v_{k}\right) & \text { if } z \in V_{k}, \\ \varepsilon^{-m / 2} u_{j}(x) & \text { if } z=(x, y) \in U_{j}\end{cases}
$$

for any $u \in \mathcal{H}^{1}\left(M_{0}\right)$. Theorem 5.3 is then implied by Lemma 2.1 in combination with the following result.

Lemma 5.4. We have $\Phi_{\varepsilon} u \in \mathcal{H}^{1}\left(M_{\varepsilon}\right)$, i.e., $\Phi_{\varepsilon}$ maps the quadratic form domain of the Laplacian on the graph into the quadratic form domain of the Laplacian on the manifold. Furthermore, for $u \in \mathcal{H}^{1}\left(M_{0}\right)$ we have

$$
\begin{aligned}
\|u\|_{M_{0}}^{2}-\left\|\Phi_{\varepsilon} u\right\|_{M_{\varepsilon}}^{2} & \leq o(1)\|u\|_{M_{0}}^{2} \\
\left\|d \Phi_{\varepsilon} u\right\|_{M_{\varepsilon}}^{2}-q_{0}(u) & =o(1) q_{0}(u) .
\end{aligned}
$$

Proof. The first assertion is true since $\Phi_{\varepsilon} u$ is constant on each thickened vertex $V_{\varepsilon, k}$ and continuous on $\partial V_{\varepsilon, k}$. Clearly, $\Phi_{\varepsilon} u$ is weakly differentiable on each thickened edge $U_{\varepsilon, j}$. Moreover, we have

$$
\begin{aligned}
& \|u\|_{M_{0}}^{2}-\left\|\Phi_{\varepsilon} u\right\|_{M_{\varepsilon}}^{2} \leq \sum_{j \in J}\left(\|u\|_{I_{j}}^{2}-\left\|\Phi_{\varepsilon} u\right\|_{U_{\varepsilon, j}}^{2}\right) \\
& =\sum_{j \in J}\left(\|u\|_{I_{j}}^{2}-(1+o(1))\left\|\Phi_{\varepsilon} u\right\|_{\tilde{U}_{\varepsilon, j}}^{2}\right)=o(1) \sum_{j \in J}\|u\|_{I_{j}}^{2}=o(1)\|u\|_{M_{0}}^{2}
\end{aligned}
$$

where we have neglected the contribution to the norm of $\Phi_{\varepsilon} u$ from the vertex parts of $M_{\varepsilon}$ and employed eqs. (4.4) and (4.8). The second relation follows from

$$
\begin{aligned}
\left\|d \Phi_{\varepsilon} u\right\|_{M_{\varepsilon}}^{2}-q_{0}(u)=\sum_{j \in J}\left((1+o(1))\left\|g_{\varepsilon}^{x x} d_{x} \Phi_{\varepsilon} u\right\|_{\tilde{U}_{\varepsilon, j}}^{2}-\left\|u^{\prime}\right\|_{I_{j}}^{2}\right) & \\
& =\sum_{j \in J}\left((1+o(1))\left\|u^{\prime}\right\|_{I_{j}}^{2}-\left\|u^{\prime}\right\|_{I_{j}}^{2}\right)=o(1) q_{0}(u)
\end{aligned}
$$

in the same way as above and with (4.5); recall that $\Phi_{\varepsilon} u$ is constant on $V_{\varepsilon, k}$ and independent of $y \in F$ on $U_{\varepsilon, j}$.

5.D. A lower bound. The reverse estimate is more difficult. Here, we will also employ averaging processes on the vertex neighbourhoods $V_{\varepsilon, k}$ which correspond to projection onto the lowest (constant) mode:

$$
C u=C_{k} u:=\frac{1}{\operatorname{vol} V_{k}} \int_{V_{k}} u d V_{k} .
$$

Recall that $V=V_{k}$ denotes the manifold $V_{k}$ with the metric $g=g_{1}$ (see Remark 5.7 for the reason why we use $V_{k}$ instead of $V_{\varepsilon, k}$ ). 
Lemma 5.5. The inequality

$$
\left|C_{k} u-N_{j} u\left(x^{0}\right)\right|^{2} \leq O\left(\varepsilon^{2 \alpha-d}\right)\|d u\|_{V_{\varepsilon, k}}^{2}
$$

holds for all $u \in \mathcal{H}^{1}\left(V_{\varepsilon, k}\right)$ where the point $x^{0}=x_{j k}^{0} \in \partial I_{j}$ corresponds to the vertex $v_{k}$.

Proof.

$$
\begin{aligned}
\left|C_{k} u-N_{j} u\left(x^{0}\right)\right|^{2} \leq \int_{F} \mid C_{k} u- & \left.u\left(x^{0}, y\right)\right|^{2} d F(y) \leq c_{1}\left(\left\|C_{k} u-u\right\|_{V_{k}}^{2}+\|d u\|_{V_{k}}^{2}\right) \\
& \leq c_{1}\left(\frac{1}{\lambda_{2}^{\mathrm{N}}\left(V_{k}\right)}+1\right)\|d u\|_{V_{k}}^{2} \leq O\left(\varepsilon^{2 \alpha-d}\right)\|d u\|_{V_{\varepsilon, k}}^{2}
\end{aligned}
$$

holds by Lemma 3.2 and Lemma 3.1] with $X=V_{k}$ and metric $g=g_{1}$, and Lemma 5.1 .

Lemma 5.6. We have

$$
\|u-C u\|_{V_{\varepsilon}}^{2} \leq O\left(\varepsilon^{\beta}\right)\|d u\|_{V_{\varepsilon}}^{2}
$$

for all $u \in \mathcal{H}^{1}\left(V_{\varepsilon}\right)$, where $\beta:=(2+d) \alpha-d$.

Proof. Using again Lemmas 3.1 and 5.1 we infer

$$
\|u-C u\|_{V_{\varepsilon}}^{2} \leq c_{1}^{+} \varepsilon^{\alpha d}\|u-C u\|_{V}^{2} \leq c_{1}^{+} \varepsilon^{\alpha d} \frac{1}{\lambda_{2}^{\mathrm{N}}(V)}\|d u\|_{V}^{2} \leq O\left(\varepsilon^{\alpha d-d+2 \alpha}\right)\|d u\|_{V_{\varepsilon}}^{2} .
$$

Notice that $\beta>0$ is equivalent to $\alpha>d /(d+2)$ and the last inequality is satisfied due to (5.2) and the fact that $d \geq 2$ holds by assumption.

Remark 5.7. For Lemma 5.6, the "natural" averaging $C_{\varepsilon} u:=\int_{V_{\varepsilon}} u d V_{\varepsilon}$ would yield the same result whereas Lemma 5.5 leads to the estimate $O\left(\varepsilon^{\beta-d}\right)$ which is worse since $2 \alpha>\beta$.

We conclude that in the fast decaying case the edge neighbourhoods lead to no spectral contribution in the limit $\varepsilon \rightarrow 0$ :

Corollary 5.8. The inequality

$$
\|u\|_{V_{\varepsilon}}^{2} \leq O\left(\varepsilon^{\alpha d-m}\right)\left(\|u\|_{U_{\varepsilon} \cup V_{\varepsilon}}^{2}+\|d u\|_{U_{\varepsilon} \cup V_{\varepsilon}}^{2}\right)
$$

holds true for all $u \in \mathcal{H}^{1}\left(U_{\varepsilon} \cup V_{\varepsilon}\right)$.

Proof. We start from the telescopic estimate

$$
\begin{gathered}
\|u\|_{V_{\varepsilon}} \leq\|u-C u\|_{V_{\varepsilon}}+\left\|C u-N u\left(x^{0}\right)\right\|_{V_{\varepsilon}}+\left\|N u\left(x^{0}\right)\right\|_{V_{\varepsilon}} \\
\leq O\left(\varepsilon^{\beta / 2}\right)\|d u\|_{V_{\varepsilon}}+\left(\operatorname{vol} V_{\varepsilon}\right)^{1 / 2}\left(O\left(\varepsilon^{(2 \alpha-d) / 2}\right)\|d u\|_{V_{\varepsilon}}^{2}+O\left(\varepsilon^{-m}\right)\left(\|u\|_{U_{\varepsilon}}^{2}+\|d u\|_{U_{\varepsilon}}^{2}\right)^{1 / 2}\right. \\
=O\left(\varepsilon^{(\alpha d-m) / 2}\right)\left(\|u\|_{U_{\varepsilon} \cup V_{\varepsilon}}^{2}+\|d u\|_{U_{\varepsilon} \cup V_{\varepsilon}}^{2}\right)^{1 / 2}
\end{gathered}
$$

where we have used Lemmas 5.6, 5.5, and 4.6, and furthermore the inequality (5.3) to obtain vol $V_{\varepsilon}=O\left(\varepsilon^{\alpha d}\right)$. Finally, note that $\beta=(d+2) \alpha-d>\alpha d-m>0$ and that $\alpha d-m>0$ is equivalent to assumption (5.2). 
Now we define the transition operator by

$$
\left(\Psi_{\varepsilon} u\right)_{j}(x):=\varepsilon^{m / 2}\left(N_{j} u(x)+\rho(x)\left(C_{k} u-N_{j} u\left(x^{0}\right)\right) \text { for } x \in I_{j k}\right.
$$

where $\rho: \mathbb{R} \longrightarrow[0,1]$ is a smooth function such that

$$
\rho\left(x^{0}\right)=1 \quad \text { and } \quad \rho(x)=0 \quad \text { for all }\left|x-x^{0}\right| \geq \frac{1}{2} \min _{j \in J} \ell_{j}
$$

where $\ell_{j}$ denotes the length of the edge $e_{j} \cong I_{j}$. Furthermore, $x^{0}=x_{j k}^{0} \in \partial I_{j}$ is the edge point which can be identified with the vertex $v_{k}$. Recall that $I_{j k}$ denotes the (closed) half of the interval $I_{j} \cong e_{j}$ adjacent with the vertex $v_{k}$ and directed away from $v_{k}$.

Lemma 5.9. We have $\Psi_{\varepsilon} u \in \mathcal{H}^{1}\left(M_{0}\right)$ if $u \in \mathcal{H}^{1}\left(M_{\varepsilon}\right)$. Furthermore,

$$
\begin{aligned}
& \|u\|_{M_{\varepsilon}}^{2}-\left\|\Psi_{\varepsilon} u\right\|_{M_{0}}^{2} \leq o(1)\left(\|u\|_{M_{\varepsilon}}^{2}+\|d u\|_{M_{\varepsilon}}^{2}\right) \\
& q_{0}\left(\Psi_{\varepsilon} u\right)-\|d u\|_{M_{\varepsilon}}^{2} \leq o(1)\left(\|u\|_{M_{\varepsilon}}^{2}+\|d u\|_{M_{\varepsilon}}^{2}\right)
\end{aligned}
$$

for all $u \in \mathcal{H}^{1}\left(M_{\varepsilon}\right)$.

Proof. The first assertion follows from $\left(\Psi_{\varepsilon} u\right)_{j}\left(x_{j k}^{0}\right)=C_{k} u$. Furthermore, we have

$$
\begin{gathered}
\|u\|_{M_{\varepsilon}}^{2}-\left\|\Psi_{\varepsilon} u\right\|_{M_{0}}^{2} \\
\leq \sum_{k \in K}\left(\|u\|_{V_{\varepsilon, k}}^{2}+\sum_{j \in J_{k}}\left(\|u\|_{U_{\varepsilon, j k}}^{2}-\varepsilon^{m}\left\|N u+\rho \cdot\left(C u-N u\left(x^{0}\right)\right)\right\|_{I_{j k}}^{2}\right)\right) \\
\leq \sum_{k \in K}\left(\|u\|_{V_{\varepsilon, k}}^{2}+\sum_{j \in J_{k}}\left(\|u\|_{U_{\varepsilon, j k}}^{2}-\left\|\varepsilon^{m / 2} N u\right\|_{I_{j k}}^{2}\right)\right. \\
\left.\quad+\sum_{j \in J_{k}}\left(\delta\left\|\varepsilon^{m / 2} N u\right\|_{I_{j k}}^{2}+\varepsilon^{m} \delta^{-1}\|\rho\|_{I_{j k}}^{2}\left|C u-N u\left(x^{0}\right)\right|^{2}\right)\right)
\end{gathered}
$$

where we have used the inequality

$$
(a+b)^{2} \geq(1-\delta) a^{2}-\frac{1}{\delta} b^{2}, \quad \delta>0
$$

The last term in the sum can be estimated by $O\left(\varepsilon^{m}\right) \delta^{-1}\left|C u-N u\left(x^{0}\right)\right|^{2}$. Applying Lemma 5.5 we arrive at the bound by $O\left(\varepsilon^{m+2 \alpha-d}\right) \delta^{-1}\left(\|u\|_{M_{\varepsilon}}^{2}+\|d u\|_{M_{\varepsilon}}^{2}\right)$. Note that $m+2 \alpha-d=2 \alpha-1>0$ since $\alpha>1 / 2$. Set $\delta:=\varepsilon^{(2 \alpha-1) / 2}$. The remaining terms can be estimated by Corollary 5.8, Lemma 4.4, and estimate (4.10).

The second inequality can be proven in the same way, namely

$$
\begin{aligned}
& q_{0}\left(\Psi_{\varepsilon} u\right)-\|d u\|_{M_{\varepsilon}}^{2} \leq \sum_{\substack{k \in K \\
j \in J_{k}}}\left(\varepsilon^{m}\left\|(N u)^{\prime}+\rho^{\prime} \cdot\left(C u-N u\left(x^{0}\right)\right)\right\|_{I_{j k}}^{2}-\|d u\|_{U_{\varepsilon, j k}}^{2}\right) \\
\leq & \sum_{\substack{k \in K \\
j \in J_{k}}}\left(\left\|\varepsilon^{m / 2}(N u)^{\prime}\right\|_{I_{j k}}^{2}-\|d u\|_{U_{\varepsilon, j k}}^{2}+\delta\left\|\varepsilon^{m / 2}(N u)^{\prime}\right\|_{I_{j k}}^{2}+\frac{2 \varepsilon^{m}}{\delta}\left\|\rho^{\prime}\right\|_{I_{j k}}^{2}\left|C u-N u\left(x^{0}\right)\right|^{2}\right)
\end{aligned}
$$


where we have used

$$
(a+b)^{2} \leq(1+\delta) a^{2}+\frac{2}{\delta} b^{2}, \quad 0<\delta \leq 1,
$$

with $\delta:=\varepsilon^{(2 \alpha-1) / 2}$. Since the norm involving $\rho^{\prime}$ is a fixed constant, the result follows from Lemma 4.5 and Lemma 5.5 .

Using Lemma [5.9 we arrive at the sought lower bound. Note that the error term $\eta_{k}$ in (2.11) can be estimated by some $\varepsilon$-independent quantity because $\lambda_{k}=$ $\lambda_{k}\left(M_{\varepsilon}\right) \leq c_{k}$ by the upper bound given in Theorem 5.3.

Theorem 5.10. We have $\lambda_{k}\left(M_{0}\right) \leq \lambda_{k}\left(M_{\varepsilon}\right)+o(1)$.

Theorem 5.2 now follows easily by combining the last result with Theorem 5.3 .

\section{Slowly DeCAying VerTex VOlume}

If the volume of the vertex region decays significantly slower than the volume of the edge neighbourhoods, the limit operator is different. At the ends of the edges we have Dirichlet boundary conditions, whereas for each vertex $v_{k}, k \in K$, we obtain an additional eigenmode. In other words, we add a point measure at each vertex to the given measure on the graph $M_{0}$; the corresponding Hilbert space and quadratic form (domain) is therefore given by

$$
\mathcal{H}_{0}:=L_{2}\left(M_{0}\right) \oplus \mathbb{C}^{K}, \quad \mathcal{D}_{0}:=\bigoplus_{j} \mathcal{H}^{1}\left(I_{j}\right) \oplus \mathbb{C}^{K}, \quad q_{0}(u):=\sum_{j}\left\|u_{j}^{\prime}\right\|_{I_{j}}^{2} .
$$

For elements of $\mathcal{H}_{0}$ we write $u=\left(\left(u_{j}\right)_{j \in J},\left(u_{k}\right)_{k \in K}\right)$ where $u_{j} \in L_{2}\left(I_{j}, p_{j}(x) d x\right)$ and $u_{k} \in \mathbb{C}$. We sometimes omit the indices and simply write $u$ instead of $u_{j}$. Note that the point contributions $u_{k}$ do not occur in the quadratic form, i.e., the additional eigenmodes have zero energy. Furthermore, the associated operator

$$
Q_{0}:=\bigoplus_{j \in J} \Delta_{I_{j}}^{\mathrm{D}} \oplus \mathbf{0}
$$

corresponds to a fully decoupled graph, i.e., a collection of independent edges, and its spectrum consists of all Dirichlet eigenvalues of the intervals $I_{j}$ and 0 . Here, $\mathbf{0}$ corresponds to the zero operator on $\mathbb{C}^{K}$.

In order to define assumptions such that a smooth metric $g_{\varepsilon}$ exists globally with different length scalings on the vertex and edge neighbourhoods, we need to introduce some additional notation (see Figure 3): let $V_{k}^{-}$be a closed submanifold of $V_{k}$ of the same dimension with a positive distance from all adjacent edge neighbourhoods $U_{j k}, j \in J_{k}$. Furthermore, we assume that the cylindrical structure of the half vertex neighbourhood $U_{j k}$ extends to the component of $V_{k} \backslash V_{k}^{-}$where $U_{j k}$ meets $V_{k}$, i.e., the closure of $V_{k} \backslash V_{k}^{-}$is diffeomorphic to the disjoint union of cylinders $[0,1] \times F$. We denote the extended cylinder containing $U_{j k}$ together with the corresponding cylindrical end (the bottle neck) of $V_{k}$ by $U_{j k}^{+}=I_{j k}^{+} \times F$ 


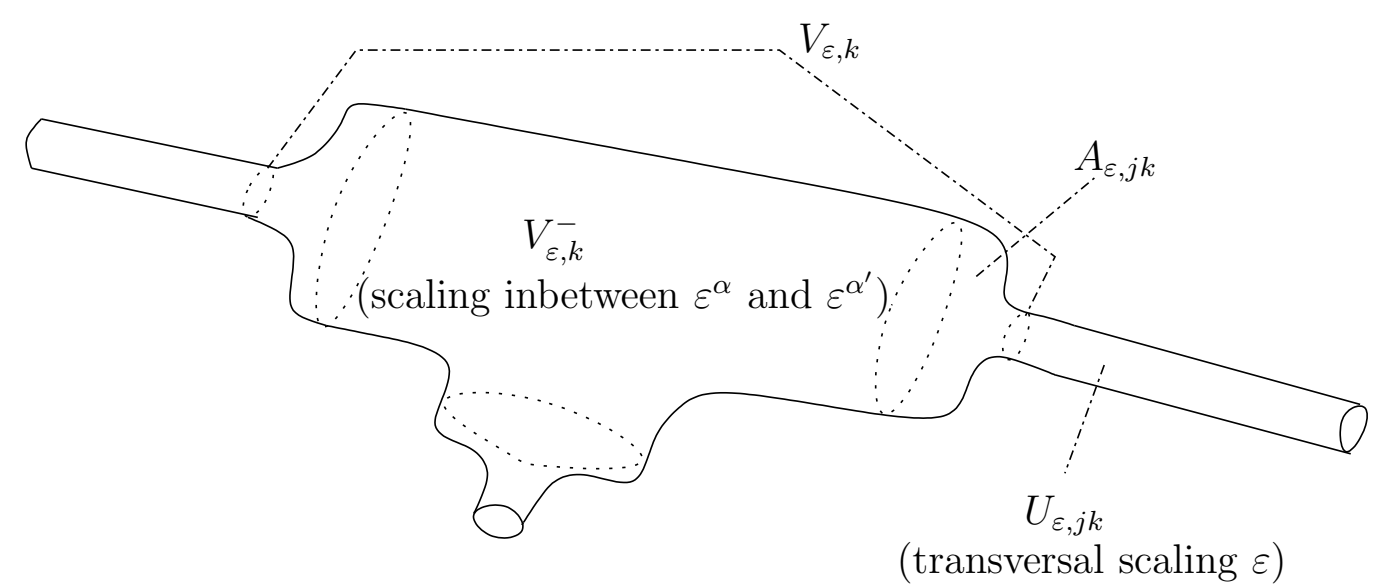

Figure 3. The decomposition with the different scaling areas.

and the bottle neck alone by $A_{j k}=I_{j k}^{0} \times F$. Note that $A_{j k}=U_{j k}^{+} \cap V_{k}$ and that $I_{j k}^{+}=I_{j} \cup I_{j k}^{0}$.

Again, we use the subscript $\varepsilon$ to indicate the corresponding Riemannian manifold with metric $g_{\varepsilon}$.

6.A. Assumption on the smaller vertex neighbourhood. We first fix the scaling behaviour on the smaller vertex neighbourhood $V_{k}^{-}$. Here, we assume that

$$
c_{-} \varepsilon^{2 \alpha} g \leq g_{\varepsilon} \leq c_{+} \varepsilon^{2 \alpha^{\prime}} g \quad \text { on } V_{k}^{-}
$$

(for the notation see (5.11) ) where

$$
0<\alpha<\frac{d-1}{d}
$$

i.e., $V_{\varepsilon, k}^{-}$scales at most as $\varepsilon^{\alpha}$ in each direction and at least as $\varepsilon^{\alpha^{\prime}}$ where

$$
\frac{d}{d+2} \alpha<\alpha^{\prime} \leq \alpha
$$

e.g., a homogeneous scaling $\left(\alpha^{\prime}=\alpha\right)$ would do. Note that $\alpha^{\prime} \leq \alpha$ is necessary in order that (6.2) makes sense whereas $\alpha d /(d+2)<\alpha^{\prime}$ ensures that the second Neumann eigenvalue of $V_{\varepsilon}^{-}$tends to $\infty$ as we will need in Lemma 6.5.

6.B. Assumptions on the bottle neck. Roughly speaking, we have to avoid that the bottle neck has more than a single neck separating $V_{\varepsilon, k}$ in more than one part as $\varepsilon \rightarrow 0$. In that case more than one zero eigenmode occur in the limit.

We use the same notation as in Section 4 for the metric $g_{\varepsilon}$ on the bottle neck $A=A_{j k}$ and set

$$
\tilde{g}_{\varepsilon}:=a_{\varepsilon}^{2}(x) d x^{2}+r_{\varepsilon}^{2}(x) h(y), \quad(x, y) \in A=I^{0} \times F
$$


for the (pure) product metric on $A$. Here, $a_{\varepsilon}=a_{\varepsilon, j k}$ and $r_{\varepsilon}=r_{\varepsilon, j k}$ are strictly positive smooth functions. Note that $r_{\varepsilon}$ defines the radius of the fibre $\{x\} \times F$ at the point $x$. Again, we denote by $G_{\varepsilon}$ and $\tilde{G}_{\varepsilon}$ the $d \times d$-matrices associated to the metrics $g_{\varepsilon}$ and $\tilde{g}_{\varepsilon}$ with respect to the coordinates $\left.(x, y) \in I^{0} \times F\right)$ and assume that the two metrics coincide up to an error term as $\varepsilon \rightarrow 0$, more specifically

$$
G_{\varepsilon}=\tilde{G}_{\varepsilon}+\left(\begin{array}{cc}
o\left(a_{\varepsilon}^{2}\right) & o\left(a_{\varepsilon} r_{\varepsilon}\right) \\
o\left(a_{\varepsilon} r_{\varepsilon}\right) & o\left(r_{\varepsilon}^{2}\right)
\end{array}\right)=\left(\begin{array}{cc}
(1+o(1)) a_{\varepsilon}^{2} & o\left(a_{\varepsilon} r_{\varepsilon}\right) \\
o\left(a_{\varepsilon} r_{\varepsilon}\right) & (H+o(1)) r_{\varepsilon}^{2}
\end{array}\right)
$$

uniformly on $A$.

We prove the following lemma in the same way as Lemma 4.3.

Lemma 6.1. Suppose that $g_{\varepsilon}, \tilde{g}_{\varepsilon}$ are given as above then

$$
\begin{aligned}
\left(\operatorname{det} G_{\varepsilon}\right)^{\frac{1}{2}} & =(1+o(1))\left(\operatorname{det} \tilde{G}_{\varepsilon}\right)^{\frac{1}{2}} \\
g_{\varepsilon}^{x x} & :=\left(G_{\varepsilon}^{-1}\right)_{x x}=a_{\varepsilon}^{-2}(1+o(1)) \\
a_{\varepsilon}^{-2}\left|d_{x} u\right|^{2} & \leq O(1)|d u|_{g_{\varepsilon}}^{2}
\end{aligned}
$$

where $d_{x}$ denotes the partial derivative with respect to $x$.

To make a smooth junction between the metrics on $U_{j}$ and $V_{k}^{-}$possible, we assume that

$$
\begin{aligned}
& a_{\varepsilon}(x)=\varepsilon^{\alpha}, \\
& r_{\varepsilon}(x)=\varepsilon^{\alpha} \\
& \text { near } x^{+} \\
& a_{\varepsilon}(x)=1, \\
& r_{\varepsilon}(x)=\varepsilon r_{-} \\
& \text {near } x^{0}
\end{aligned}
$$

where $x \in I^{0}=\left[x^{+}, x^{0}\right]$ and $r_{-}:=r_{j}\left(x^{0}\right)$ (the radius of the fibre at $x^{0}$, see also equation (4.1)).

Furthermore, we assume that

$$
a_{\varepsilon}(x) \leq\left\{\begin{array}{ll}
\varepsilon^{\alpha} & \text { on }\left[x^{+}, x^{0}-\delta_{0},\right] \\
1 & \text { on }\left[x^{0}-\delta_{0}, x^{0}\right]
\end{array} \quad \varepsilon r_{-} \leq r_{\varepsilon}(x) \leq \begin{cases}\varepsilon^{\alpha} & \text { on }\left[x^{+}, x^{+}+\delta_{+}\right] \\
\varepsilon r_{+} & \text {on }\left[x^{+}+\delta_{+}, x^{0}\right]\end{cases}\right.
$$

for some constant $r_{+} \geq r_{-}$, where $\delta_{0}=\varepsilon^{\alpha}$ and $\delta_{+}=\varepsilon^{(1-\alpha) m}=\varepsilon^{\alpha} \varepsilon^{m-\alpha d}$. These
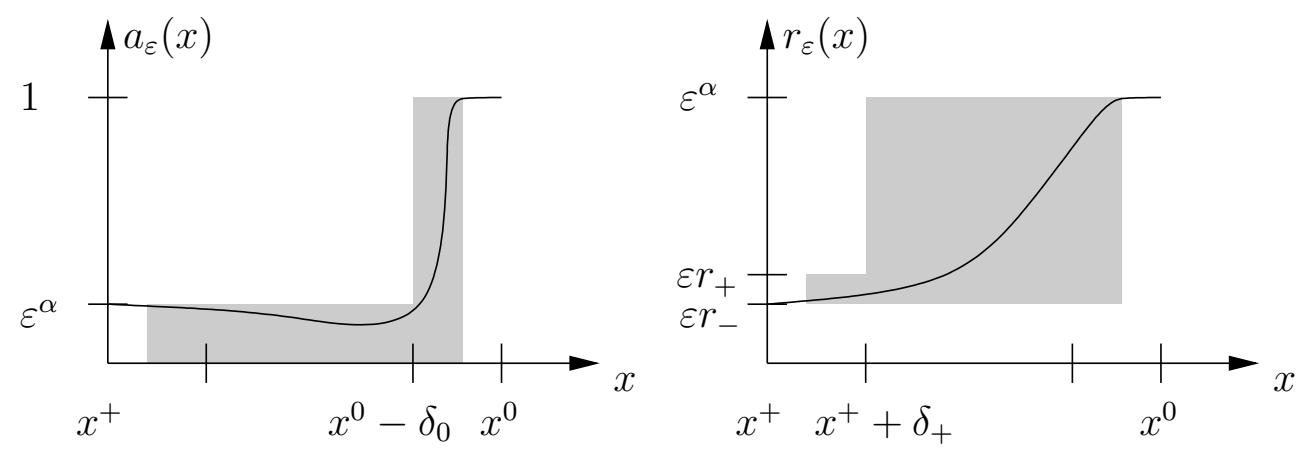

FIGURE 4. The functions $a_{\varepsilon}$ and $r_{\varepsilon}$ in its allowed range (in grey). 
assumptions are needed in Lemma 6.7, e.g. to assure that the eigenfunctions of $M_{\varepsilon}$ do not concentrate on $A_{\varepsilon, j k}$ (i.e., (6.18) holds).

6.C. Convergence of the spectra. With the above prerequisites we can finally formulate the main result of this section:

Theorem 6.2. Under the stated assumptions $\lambda_{k}\left(M_{\varepsilon}\right) \rightarrow \lambda_{k}\left(Q_{0}\right)$ as $\varepsilon \rightarrow 0$. More precisely, the first $|K|$ eigenvalues tend to 0 , while the remaining bounded eigenvalue branches tend to Dirichlet eigenvalues of the intervals $I_{j}$, i.e.,

$$
\begin{array}{lrl}
\lambda_{k}\left(M_{\varepsilon}\right) & \rightarrow 0 & \text { if } 1 \leq k \leq|K| \\
\lambda_{k}\left(M_{\varepsilon}\right) \rightarrow \lambda_{k-|K|}^{\mathrm{D}}\left(\dot{\cup}_{j \in J} I_{j}\right) & \text { if } k>|K|,
\end{array}
$$

where $\lambda_{n}^{\mathrm{D}}\left(\bigcup_{j \in J} I_{j}\right)$ denotes the Dirichlet eigenvalues $\lambda_{l}^{\mathrm{D}}\left(\Delta_{I_{j}}\right)$ of the operators on $I_{j}(j \in J)$ defined as in (2.2), reordered with respect to multiplicity. In particular, if the length of all the edges $I_{j}$ is $\ell$ and $p_{j}(x)=1$ for all $j$, we have

$$
\lambda_{k}\left(M_{\varepsilon}\right) \rightarrow \lambda_{m}^{\mathrm{D}}([0, \ell])=\pi^{2} m^{2} / \ell^{2} \quad \text { if } k=(m-1)|J|+1, \ldots, m|J| .
$$

Again, our aim is to find a two sided estimate on each eigenvalue $\lambda_{k}\left(M_{\varepsilon}\right)$ by means of $\lambda_{k}\left(Q_{0}\right)$ with an error which is $o(1)$ w.r.t. the parameter $\varepsilon$.

6.D. An upper bound. The following upper eigenvalue estimate is slightly more difficult to show than in the previous section:

Theorem 6.3. $\lambda_{k}\left(M_{\varepsilon}\right) \leq \lambda_{k}\left(Q_{0}\right)+o(1)$ holds as $\varepsilon \rightarrow 0$.

To prove it, we define the transition operator by

$$
\Phi_{\varepsilon} u(z):= \begin{cases}\left(\operatorname{vol} V_{\varepsilon, k}^{-}\right)^{-1 / 2} u_{k} & \text { if } z \in V_{k}, \\ \varepsilon^{-m / 2} u_{j}(x)+\left(\operatorname{vol} V_{\varepsilon, k}^{-}\right)^{-1 / 2} \rho(x) u_{k} & \text { if } z=(x, y) \in U_{j}\end{cases}
$$

for any $u \in \mathcal{D}_{0}$, where $\rho$ is a smooth function as in (5.11) and $x^{0}=x_{j k}^{0}$ denotes the endpoint of the half-edge $I_{j k}$ corresponding to the vertex $v_{k}$. Theorem 6.3 is then implied by Lemma 2.1] in combination with the following result.

Lemma 6.4. We have $\Phi_{\varepsilon} u \in \mathcal{H}^{1}\left(M_{\varepsilon}\right)$, i.e., $\Phi_{\varepsilon}$ maps the quadratic form domain $\mathcal{D}_{0}$ into the quadratic form domain of the Laplacian on the manifold. Furthermore,

$$
\begin{aligned}
\|u\|_{\mathcal{H}_{0}}^{2}-\left\|\Phi_{\varepsilon} u\right\|_{M_{\varepsilon}}^{2} & \leq o(1)\|u\|_{\mathcal{H}_{0}}^{2} \\
\left\|d \Phi_{\varepsilon} u\right\|_{M_{\varepsilon}}^{2}-q_{0}(u) & \leq o(1)\left(\|u\|_{\mathcal{H}_{0}}^{2}+q_{0}(u)\right)
\end{aligned}
$$

Proof. Since $u_{j} \uparrow_{\partial I_{j}}=0$, the function $\Phi_{\varepsilon} u$ agrees on $\partial V_{\varepsilon, k}$ for both definitions. Clearly, $\Phi_{\varepsilon} u$ is weakly differentiable on each thickened edge $U_{\varepsilon, j}$. Moreover, we 
have

$$
\begin{aligned}
\|u\|_{\mathcal{H}_{0}}^{2}-\left\|\Phi_{\varepsilon} u\right\|_{M_{\varepsilon}}^{2} \leq \sum_{\substack{k \in K \\
j \in J_{k}}}\left(\left(\|u\|_{I_{j k}}^{2}-(1+o(1)) \| \varepsilon^{-\frac{m}{2}} u\right.\right. & \left.+\left(\operatorname{vol} V_{\varepsilon, k}^{-}\right)^{-\frac{1}{2}} \rho u_{k} \|_{\tilde{U}_{\varepsilon, j k}}^{2}\right)+ \\
& \left.+\left(\left|u_{k}\right|^{2}-\left\|\Phi_{\varepsilon} u\right\|_{V_{\varepsilon, k}^{-}}^{2}\right)\right)
\end{aligned}
$$

where we have used equation (4.4) and that $V_{\varepsilon, k}^{-} \subset V_{\varepsilon, k}$. Note that the latter sum in the last line is equal to 0 . To estimate the remaining sum, remember that $\Phi_{\varepsilon} u$ is independent of $y$ on $U_{\varepsilon, j k}$. Therefore we can apply equation (4.8), and inequality (5.14) with $\delta:=\varepsilon^{(m-\alpha d) / 2}$ yields the upper estimate

$$
\sum_{k \in K, j \in J_{k}}\left((\delta+o(1))\|u\|_{I_{j k}}^{2}+\frac{O\left(\varepsilon^{m-\alpha d}\right)}{\delta}\left|u_{k}\right|^{2}\right)=o(1)\|u\|_{\mathcal{H}_{0}}^{2} .
$$

In the last inequality, we have used the estimate $\left(\operatorname{vol} V_{\varepsilon, k}^{-}\right)^{-1} \leq O\left(\varepsilon^{-\alpha d}\right)$ which follows from the lower bound of (6.2). Note that $\delta=o(1)$ by assumption (6.3). The second relation follows from

$$
\begin{array}{r}
\left\|d \Phi_{\varepsilon} u\right\|_{M_{\varepsilon}}^{2}-q_{0}(u)=\sum_{\substack{k \in K \\
j \in J_{k}}}\left((1+o(1)) \varepsilon^{m}\left\|\varepsilon^{-\frac{m}{2}} u^{\prime}+\left(\operatorname{vol} V_{\varepsilon, k}^{-}\right)^{-\frac{1}{2}} \rho^{\prime} u_{k}\right\|_{I_{j k}}^{2}-\left\|u^{\prime}\right\|_{I_{j k}}^{2}\right) \\
\leq \sum_{\substack{k \in K \\
j \in J_{k}}}(1+o(1))\left(\delta\left\|u^{\prime}\right\|_{I_{j k}}^{2}+\frac{2 \varepsilon^{m}}{\delta \operatorname{vol} V_{\varepsilon, k}^{-}}\left\|\rho^{\prime}\right\|_{I_{j k}}^{2}\left|u_{k}\right|^{2}\right) \leq o(1)\left(\|u\|_{\mathcal{H}_{0}}^{2}+q_{0}(u)\right)
\end{array}
$$

in the same way as above together with (4.5) for the second equality and (5.15) in the last line; recall that $\Phi_{\varepsilon} u$ is constant on $V_{\varepsilon, k}$.

Note that we need a counterpart to $\|u\|_{V_{\varepsilon, k}}^{2}$ on the limit Hilbert space $\mathcal{H}_{0}$. In the case of a fast decaying vertex volume in the previous section, the corresponding norm vanished (see Corollary [5.8), but here we need the additional subspace $\mathbb{C}^{K}$ in $\mathcal{H}_{0}$ coming from extra point measures at the vertices.

Furthermore, note that the upper bound estimate on $\lambda_{k}\left(M_{\varepsilon}\right)$ already proven in Lemma 5.4 remains valid in this setting, but it is too rough for the present purpose.

6.E. A lower bound. Again, the reverse estimate is more difficult. We will employ averaging processes also on the vertex neighbourhoods; this time with the $\varepsilon$-scaled manifold $V_{\varepsilon, k}^{-}$:

$$
C_{\varepsilon}^{-} u=C_{\varepsilon, k}^{-} u:=\frac{1}{\operatorname{vol} V_{\varepsilon, k}^{-}} \int_{V_{\varepsilon, k}^{-}} u d V_{\varepsilon, k}^{-} .
$$

In the first lemma, we prove an estimate similar to the one in Lemma [5.6. Note that $\left\|C_{\varepsilon}^{-} u\right\| \leq\|u\|$ by Cauchy-Schwarz, but we need the reverse inequality. 
Lemma 6.5. For any $u \in \mathcal{H}^{1}\left(V_{\varepsilon}^{-}\right)$we have

$$
\|u\|_{V_{\varepsilon}^{-}}^{2}-\left\|C_{\varepsilon}^{-} u\right\|_{V_{\varepsilon}^{-}}^{2} \leq o(1)\left(\|u\|_{V_{\varepsilon}^{-}}^{2}+\|d u\|_{V_{\varepsilon}^{-}}^{2}\right) .
$$

Proof. Apply Lemma 3.1 with $X=V_{\varepsilon}^{-}$and $\delta=\varepsilon^{\left((d+2) \alpha^{\prime}-d \alpha\right) / 2}$. From the minmax principle we obtain $\lambda_{2}^{\mathrm{N}}\left(V_{\varepsilon}^{-}\right) \geq O\left(\varepsilon^{d\left(\alpha-\alpha^{\prime}\right)-2 \alpha^{\prime}}\right)$. Note that $\delta=o(1)$ since $(d+2) \alpha^{\prime}-d \alpha>0$ by (6.4).

The next three results are valid independently of the assumptions on $\alpha$ given in (6.3) and (6.4). We will need these results also for the borderline case $\alpha=$ $(d-1) / d$ in the next section.

We need an estimate on the average $\left|N u\left(x^{0}\right)\right|^{2}$. Since on the bottle neck $A_{\varepsilon, j k}$, the estimates are quite delicate, we first prove the result for $\left|N u\left(x^{+}\right)\right|^{2}$ (i.e., on $\partial V_{\varepsilon, k}^{-}$where the scaling of the metric is of the right order. The error is controlled by (6.17). Note that this estimate is a counterpart to the estimate in Lemma 4.6 where we extended the function to the edge neighbourhood $U_{\varepsilon, j}$ (useful in the case of fast decaying vertex volume, $\alpha d-m>0$ ). This is not possible here, since $\alpha d-m<0$. Therefore, we extend the function to the vertex neighbourhood $V_{\varepsilon, k}^{-}$.

Lemma 6.6. The inequality

$$
\left|N u\left(x^{+}\right)\right|^{2} \leq\left\|u\left(x^{+}, \cdot\right)\right\|_{F}^{2} \leq O\left(\varepsilon^{-\alpha d}\right)\left(\|u\|_{V_{\varepsilon}^{-}}^{2}+\|d u\|_{V_{\varepsilon}^{-}}^{2}\right)
$$

holds for any $u \in \mathcal{H}^{1}\left(V_{\varepsilon}^{-}\right)$.

Proof. We have

$$
\begin{aligned}
& \left|N u\left(x^{+}\right)\right|^{2} \leq \int_{F}\left|u\left(x^{+}, y\right)\right|^{2} d F(y) \\
& \quad \leq c_{1}\left(\|u\|_{V^{-}}^{2}+\|d u\|_{V^{-}}^{2}\right) \leq O\left(\varepsilon^{-\alpha d}\right)\left(\|u\|_{V_{\varepsilon}^{-}}^{2}+\|d u\|_{V_{\varepsilon}^{-}}^{2}\right)
\end{aligned}
$$

by Lemma 3.2 with $X=V^{-}$and the lower bound in assumption (6.3).

The next lemma is the key ingredient in dealing with the bottle neck. Here, we prove two Poincaré-like estimates. Since we want to avoid a cut-off function (leading to divergent terms when being differentiated) we only prove an estimate on the difference and not on $N u\left(x^{0}\right)$ itself in (6.17). For the same reason, an integral over $F$ remains in (6.18). Note that $I_{\varepsilon}^{+}\left(x^{0}\right)=\operatorname{vol} A_{\varepsilon}$.

Lemma 6.7. There is a constant $C>0$ such that

$$
\begin{gathered}
\left|N u\left(x^{0}\right)-N u\left(x^{+}\right)\right|^{2} \leq C I_{\varepsilon}^{-}\left(x^{0}\right)\|d u\|_{A_{\varepsilon}}^{2}, \\
\|u\|_{A_{\varepsilon}}^{2} \leq 4 I_{\varepsilon}^{+}\left(x^{0}\right)\left\|u\left(x^{+}, \cdot\right)\right\|_{F}^{2}+4 C I_{\varepsilon}^{+-}\|d u\|_{A_{\varepsilon}}^{2}
\end{gathered}
$$

for all $u \in \mathcal{H}^{1}\left(A_{\varepsilon}\right)$ where

$$
I_{\varepsilon}^{ \pm}(x):=\int_{x+}^{x} a_{\varepsilon}\left(x^{\prime}\right) r_{\varepsilon}^{ \pm m}\left(x^{\prime}\right) d x^{\prime} \quad \text { and } \quad I_{\varepsilon}^{+-}:=\int_{x^{+}}^{x^{0}} a_{\varepsilon}(x) r_{\varepsilon}^{m}(x) I_{\varepsilon}^{-}(x) d x \text {. }
$$


Furthermore, under the assumption (6.10), we have

$$
I_{\varepsilon}^{-}\left(x^{0}\right)=o\left(\varepsilon^{-m}\right), \quad I_{\varepsilon}^{+}\left(x^{0}\right)=o\left(\varepsilon^{\alpha d}\right) \quad \text { and } \quad I_{\varepsilon}^{+-}=o(1) .
$$

Proof. For a smooth function $u$ we have

$$
u(x, y)-u\left(x^{+}, y\right)=\int_{x^{+}}^{x} \partial_{x} u\left(x^{\prime}, y\right) d x^{\prime} .
$$

For the first assertion, we set $x=x^{0}$, foremost integrate over $y \in F$ and then apply Cauchy-Schwarz

$$
\begin{aligned}
\left|N u\left(x^{0}\right)-N u\left(x^{+}\right)\right|^{2} \leq \int_{F} & \int_{x^{+}}^{x^{0}} a_{\varepsilon}^{2}\left(x^{\prime}\right) \operatorname{det} G_{\varepsilon}\left(x^{\prime}, y\right)^{-\frac{1}{2}} d x^{\prime} \times \\
& \times \int_{x^{+}}^{x^{0}} a_{\varepsilon}^{-2}\left(x^{\prime}\right)\left|\partial_{x} u\left(x^{\prime}, y\right)\right|^{2} \operatorname{det} G_{\varepsilon}\left(x^{\prime}, y\right)^{\frac{1}{2}} d x^{\prime} d F(y) .
\end{aligned}
$$

The first integrand over $x^{\prime}$ can be estimated by $C a_{\varepsilon}\left(x^{\prime}\right) r_{\varepsilon}^{-m}$ applying (6.7). Therefore, the first integral is smaller than $C I_{\varepsilon}^{-}\left(x^{0}\right)$. The second integral together with the integral over $F$ can be estimated by $O(1)\|d u\|_{A_{\varepsilon}}^{2}$ applying (6.9).

For the second assertion, we first apply Cauchy-Schwarz (and (5.15) with $\delta=1$ ) to (6.19) and than integrate over $y \in F$ to obtain

$$
\begin{aligned}
\int_{F}|u(x, y)|^{2} d F(y) \leq & 2 \int_{F}\left|u\left(x^{+}, y\right)\right|^{2} d F(y)+2 \int_{F} \int_{x^{+}}^{x} a_{\varepsilon}^{2}\left(x^{\prime}\right) \operatorname{det} G\left(x^{\prime}, y\right)^{-\frac{1}{2}} d x^{\prime} \times \\
& \times \int_{x^{+}}^{x} a_{\varepsilon}^{-2}\left(x^{\prime}\right)\left|\partial_{x} u\left(x^{\prime}, y\right)\right|^{2} \operatorname{det} G\left(x^{\prime}, y\right)^{\frac{1}{2}} d x^{\prime} d F(y) .
\end{aligned}
$$

The first integral over $x^{\prime}$ can be estimated as before by $C I_{\varepsilon}^{-}(x)$. Finally, multiplying with $a_{\varepsilon}(x) r_{\varepsilon}^{m}(x)$ and integrating over $x \in I^{0}$ yields

$$
\|u\|_{\tilde{A}_{\varepsilon}}^{2} \leq 2 I_{\varepsilon}^{+}\left(x_{0}\right)\left\|u\left(x^{+}, \cdot\right)\right\|_{F}^{2}+2 C I_{\varepsilon}^{+-}\|d u\|_{A_{\varepsilon}}^{2} .
$$

Applying (6.7) once more we obtain the desired estimate over $A_{\varepsilon}$ instead of $\tilde{A}_{\varepsilon}$ (note that $2 /(1+o(1)) \leq 4$ provided $\varepsilon$ is small enough). The general case of non-smooth functions can easily shown with approximation arguments.

The integral estimates follow from

$$
I_{\varepsilon}^{-}\left(x^{0}\right) \leq \int_{x^{+}}^{x^{0}-\delta_{0}} \varepsilon^{\alpha}\left(\varepsilon r_{-}\right)^{-m} d x+\int_{x^{0}-\delta_{0}}^{x^{0}}\left(\varepsilon r_{-}\right)^{-m} d x=\left(\varepsilon^{\alpha}+\delta_{0}\right) O\left(\varepsilon^{-m}\right) .
$$

Since $\delta_{0}=\varepsilon^{\alpha}$, we have $I_{\varepsilon}^{-}\left(x^{0}\right) \leq O\left(\varepsilon^{\alpha-m}\right)$. Next, we have

$$
\begin{aligned}
I_{\varepsilon}^{+}\left(x^{0}\right) \leq \int_{x^{+}}^{x^{+}+\delta_{+}} \varepsilon^{\alpha} \varepsilon^{\alpha m} d x & +\int_{x^{+}+\delta_{+}}^{x^{0}-\delta_{0}} \varepsilon^{\alpha}\left(\varepsilon r_{+}\right)^{m} d x \\
& +\int_{x^{0}-\delta_{0}}^{x^{0}}\left(\varepsilon r_{+}\right)^{m} d x=\delta_{+} O\left(\varepsilon^{\alpha d}\right)+\left(\varepsilon^{\alpha}+\delta_{0}\right) O\left(\varepsilon^{m}\right)
\end{aligned}
$$


and therefore $I_{\varepsilon}^{+}\left(x^{0}\right) \leq O\left(\varepsilon^{\alpha+m}\right)=O\left(\varepsilon^{\alpha+m-\alpha d}\right) O\left(\varepsilon^{\alpha d}\right)$ since $\delta_{+}=\varepsilon^{\alpha} \varepsilon^{m-\alpha d}$. The last assertion follows from $I_{\varepsilon}^{+-} \leq I_{\varepsilon}^{-}\left(x_{0}\right) I_{\varepsilon}^{+}\left(x_{0}\right) \leq O\left(\varepsilon^{2 \alpha}\right)$.

The following corollary is again independent of the assumption we made about $\alpha$ in (6.3) and (6.4), in particular, it is also valid in the setting of the borderline case of Section 7.

Corollary 6.8. For all $u \in \mathcal{H}^{1}\left(V_{\varepsilon}\right)$ we have

$$
\|u\|_{A_{\varepsilon}}^{2} \leq o(1)\left(\|u\|_{V_{\varepsilon}}^{2}+\|d u\|_{V_{\varepsilon}}^{2}\right) \text {. }
$$

Proof. We only have to put together (6.18) and Lemma 6.6.

We now formulate a consequence of the preceding lemmas under the assumption (6.3).

Corollary 6.9. Suppose $0<\alpha<m / d=(d-1) / d$. Then we have

$$
\left|N u\left(x^{0}\right)\right|^{2} \leq o\left(\varepsilon^{-m}\right)\left(\|u\|_{V_{\varepsilon}}^{2}+\|d u\|_{V_{\varepsilon}}^{2}\right)
$$

for all $u \in \mathcal{H}^{1}\left(V_{\varepsilon}\right)$.

Proof. Applying (5.14) with $\delta=1 / 2$ to (6.17) we obtain

$$
\left|N u\left(x^{0}\right)\right|^{2} \leq o\left(\varepsilon^{-m}\right)\|d u\|_{A_{\varepsilon}}^{2}+4\left|N u\left(x^{+}\right)\right|^{2} .
$$

The second term is of order $O\left(\varepsilon^{-\alpha d}\right)$ by Lemma 6.6 and therefore also of order $o\left(\varepsilon^{-m}\right)$ by the assumption on $\alpha$.

In this section, we define the transition operator by

$$
\begin{aligned}
\left(\Psi_{\varepsilon} u\right)_{j}(x) & :=\varepsilon^{m / 2} N_{j} u(x)-\rho(x) N_{j} u\left(x^{0}\right) \text { for } x \in I_{j k} \\
\left(\Psi_{\varepsilon} u\right)_{k} & :=\left(\operatorname{vol} V_{\varepsilon, k}^{-}\right)^{1 / 2} C_{\varepsilon, k}^{-} u
\end{aligned}
$$

where $\rho$ is a smooth function as in (5.11) and $x^{0}=x_{j k}^{0}$ denotes the endpoint of the half-edge $I_{j k}$ corresponding to the vertex $v_{k}$.

Lemma 6.10. We have $\Psi_{\varepsilon} u \in \mathcal{D}_{0}$ if $u \in \mathcal{H}^{1}\left(M_{\varepsilon}\right)$. Furthermore,

$$
\begin{gathered}
\|u\|_{M_{\varepsilon}}^{2}-\left\|\Psi_{\varepsilon} u\right\|_{\mathcal{H}_{0}}^{2} \leq o(1)\left(\|u\|_{M_{\varepsilon}}^{2}+\|d u\|_{M_{\varepsilon}}^{2}\right) \\
q_{0}\left(\Psi_{\varepsilon} u\right)-\|d u\|_{M_{\varepsilon}}^{2} \leq o(1)\left(\|u\|_{M_{\varepsilon}}^{2}+\|d u\|_{M_{\varepsilon}}^{2}\right)
\end{gathered}
$$

for all $u \in \mathcal{H}^{1}\left(M_{\varepsilon}\right)$.

Proof. The first assertion follows from the fact that $\left(\Psi_{\varepsilon} u\right)_{j}\left(x^{0}\right)=0$. Furthermore, we have

$$
\begin{aligned}
\|u\|_{M_{\varepsilon}}^{2}-\left\|\Psi_{\varepsilon} u\right\|_{\mathcal{H}_{0}}^{2} \leq & \sum_{k \in K}\left(\left(\|u\|_{V_{\varepsilon, k}^{-}}^{2}-\left\|C_{\varepsilon}^{-} u\right\|_{V_{\varepsilon, k}^{-}}^{2}\right)\right. \\
& \left.+\sum_{j \in J_{k}}\left(\|u\|_{A_{\varepsilon, j k}}^{2}+\|u\|_{U_{\varepsilon, j k}}^{2}-\varepsilon^{m}\left\|N u-\rho \cdot N u\left(x^{0}\right)\right\|_{I_{j k}}^{2}\right)\right) .
\end{aligned}
$$


The first difference is of the desired form by Lemma 6.5. Furthermore, the integral over the "bottle necks" $A_{\varepsilon, j k}$ can be estimated in the needed way by Corollary 6.8, Applying (15.14) to the remaining difference in the last sum we obtain the upper estimate by

$$
\left(\|u\|_{U_{\varepsilon, j k}}^{2}-\varepsilon^{m}\|N u\|_{I_{j k}}^{2}\right)+\delta \varepsilon^{m}\|N u\|_{I_{j k}}^{2}+\frac{\varepsilon^{m}}{\delta}\|\rho\|_{I_{j k}}^{2}\left|N u\left(x^{0}\right)\right|^{2}
$$

For the first two terms we obtain the sought bound by virtue of Lemma 4.4 and (4.10); for the remaining term one has to apply Corollary 6.9.

The second inequality can be proven in the same way, namely

$$
\begin{aligned}
q_{0}\left(\Psi_{\varepsilon} u\right)- & \|d u\|_{M_{\varepsilon}}^{2} \\
& =\sum_{k \in K}\left(-\|d u\|_{V_{\varepsilon, k}^{-}}^{2}+\sum_{j \in J_{k}}\left(\varepsilon^{m}\left\|(N u)^{\prime}-\rho^{\prime} N u\left(x^{0}\right)\right\|_{I_{j k}}^{2}-\|d u\|_{U_{\varepsilon, j k}}^{2}\right)\right)
\end{aligned}
$$

We omit the norm contribution from $V_{\varepsilon, k}^{-}$and estimate the remaining difference with (5.15) and obtain (up to the summation)

$$
\left(\varepsilon^{m}\left\|(N u)^{\prime}\right\|_{I_{j k}}^{2}-\|d u\|_{U_{\varepsilon, j k}}^{2}\right)+\delta \varepsilon^{m}\left\|(N u)^{\prime}\right\|_{I_{j k}}^{2}+2 \frac{\varepsilon^{m}}{\delta}\left\|\rho^{\prime}\right\|_{I_{j k}}^{2}\left|N u\left(x^{0}\right)\right|^{2} .
$$

For the first difference we obtain the needed estimate by virtue of Lemma 4.5 . An upper bound for the remaining term is of the same form as before.

Using Lemma 6.10 we arrive at the sought lower bound. Note that the error term $\eta_{k}$ in (2.11) can be estimated by some $\varepsilon$-independent quantity because $\lambda_{k}=$ $\lambda_{k}\left(M_{\varepsilon}\right) \leq c_{k}$ by Theorem 6.3 .

Theorem 6.11. We have $\lambda_{k}\left(Q_{0}\right) \leq \lambda_{k}\left(M_{\varepsilon}\right)+o(1)$.

Theorem 6.2 now follows easily by combining the last result with Theorem 6.3.

\section{THE BORDERLINE CASE}

7.A. Definition of the thickened vertices. If the volume of the vertex region decays at the same rate as the volume of the edge neighbourhoods, the limit operator acts again in the extended Hilbert space introduced in the previous section but it is not decoupled anymore. Thus it is not supported by the graph alone, in particular, it is not the Hamiltonian with the boundary conditions (2.4).

We start with the definition of the limit operator. The corresponding Hilbert space and quadratic form are given by

$$
\mathcal{H}_{0}:=L_{2}\left(M_{0}\right) \oplus \mathbb{C}^{K}, \quad q_{0}(u):=\sum_{j}\left\|u_{j}^{\prime}\right\|_{I_{j}}^{2},
$$

where the form domain $\mathcal{D}_{0}$ of $q_{0}$ is given by those functions $u=\left(\left(u_{j}\right)_{j \in J},\left(u_{k}\right)_{k \in K}\right)$ such that

$$
u \in \mathcal{H}^{1}\left(M_{0}\right) \oplus \mathbb{C}^{K} \quad \text { and } \quad\left(\operatorname{vol} V_{k}^{-}\right)^{1 / 2} u_{j}\left(v_{k}\right)=u_{k}
$$


for all $j \in J_{k}$ and $k \in K$, i.e., values of the functions at the edge endpoints $v_{k} \equiv x_{j k}^{0}$ are now coupled with the additional wave function components; recall that $V_{k}^{-}$denotes the manifold $V_{\varepsilon, k}^{-}$with $\varepsilon=1$. The corresponding operator $Q_{0}$ is given by

$$
Q_{0} u=\left(\left(-\frac{1}{p_{j}}\left(p_{j} u_{j}^{\prime}\right)^{\prime}\right)_{j},\left(-\left(\operatorname{vol} V_{k}^{-}\right)^{-\frac{1}{2}} \sum_{j \in J_{k}} p_{j}\left(v_{k}\right) u_{j}^{\prime}\left(v_{k}\right)\right)_{k}\right)
$$

it depends parametrically on $\operatorname{vol}\left(V_{k}^{-}\right)$but we refrain from marking this fact explicitly. Again, this operator has a purely discrete spectrum provided the graph $M_{0}$ is finite.

As we have said, $Q_{0}$ is not a graph operator with the conditions (2.4). Nevertheless, there is a similarity between the two noticed by Kuchment and Zeng in KuZ03. To solve the spectral problem $Q_{0} u=\lambda u$ one has to find $\left(u_{j}\right)_{j \in J}$ such that $-\left(p_{j} u_{j}^{\prime}\right)^{\prime} / p_{j}=\lambda u_{j}$ and at the vertices the functions satisfy the conditions

$$
\sum_{j \in J_{k}} p_{j}\left(v_{k}\right) u_{j}^{\prime}\left(v_{k}\right)=-\lambda\left(\operatorname{vol} V_{k}^{-}\right) u\left(v_{k}\right)
$$

This looks like (2.4), the difference is that the coefficient at the right-hand side is not a constant but a multiple of the spectral parameter; in physical terms one may say that the coupling strength at a vertex is proportional to the energy.

After this digression let us return to the limiting properties. We adopt again the assumption (6.10) in this section. Instead of (6.2) we suppose now that on the vertex neighbourhood the metric satisfies the relation

$$
g_{\varepsilon}=\varepsilon^{2 \alpha} g+o\left(\varepsilon^{2 \alpha}\right) \quad \text { on } V_{k}^{-}
$$

with

$$
\alpha=\frac{d-1}{d}
$$

which corresponds to the above mentioned equal decay rate for the volume of the edge and vertex neighbourhoods. In particular, we have

$$
\|u\|_{V_{\varepsilon}^{-}}^{2}=\varepsilon^{\alpha d}(1+o(1))\|u\|_{V^{-}}^{2} \quad \text { and } \quad\|d u\|_{V_{\varepsilon}^{-}}^{2}=\varepsilon^{\alpha(d-2)}(1+o(1))\|d u\|_{V^{-}}^{2}
$$

and

$$
\operatorname{vol}\left(V_{\varepsilon}^{-}\right)=\varepsilon^{\alpha d}(1+o(1)) \operatorname{vol}\left(V^{-}\right)
$$

for each $V^{-}=V_{k}^{-}$as in Lemmas 4.3 and 5.1 .

7.B. Convergence of the spectra. With the above prerequisites we can finally formulate the main result of this section:

Theorem 7.1. Under the stated assumptions $\lambda_{k}\left(M_{\varepsilon}\right) \rightarrow \lambda_{k}\left(Q_{0}\right)$ as $\varepsilon \rightarrow 0$.

To prove it, our aim is again to find a two sided estimate on each eigenvalue $\lambda_{k}\left(M_{\varepsilon}\right)$ by means of $\lambda_{k}\left(Q_{0}\right)$ with an error which is $o(1)$ w.r.t. the parameter $\varepsilon$. 
7.C. An upper bound. Again, we first show the easier upper eigenvalue estimate:

Theorem 7.2. $\lambda_{k}\left(M_{\varepsilon}\right) \leq \lambda_{k}\left(Q_{0}\right)+o(1)$ holds as $\varepsilon \rightarrow 0$.

We define the transition operator by

$$
\Phi_{\varepsilon} u(z):= \begin{cases}\operatorname{vol}\left(V_{\varepsilon, k}^{-}\right)^{-1 / 2} u_{k} & \text { if } z \in V_{k}, \\ \varepsilon^{-m / 2} u_{j}(x)+\rho(x) \times & \text { if } z=(x, y) \in U_{j} \\ \times\left(\operatorname{vol}\left(V_{\varepsilon, k}^{-}\right)^{-1 / 2} u_{k}-\varepsilon^{-m / 2} u_{j}\left(x^{0}\right)\right) & \end{cases}
$$

for any $u \in \mathcal{D}_{0}$, where $\rho$ is a smooth function as in (5.11) and $x^{0}=x_{j k}^{0}$ denotes the endpoint of the half-edge $I_{j k}$ away from the vertex $v_{k}$. Theorem 7.2 is then implied by Lemma 2.1 in combination with the following result.

Lemma 7.3. We have $\Phi_{\varepsilon} u \in \mathcal{H}^{1}\left(M_{\varepsilon}\right)$, i.e., $\Phi_{\varepsilon}$ maps the quadratic form domain $\mathcal{D}_{0}$ into the quadratic form domain of the Laplacian on the manifold. Furthermore,

$$
\begin{aligned}
\|u\|_{\mathcal{H}_{0}}^{2}-\left\|\Phi_{\varepsilon} u\right\|_{M_{\varepsilon}}^{2} & \leq o(1)\|u\|_{\mathcal{H}_{0}}^{2} \\
\left\|d \Phi_{\varepsilon} u\right\|_{M_{\varepsilon}}^{2}-q_{0}(u) & \leq o(1)\left(\|u\|_{\mathcal{H}_{0}}^{2}+q_{0}(u)\right)
\end{aligned}
$$

Proof. The argument is analogous to the proof of Lemma 6.4. The only difference is that we need the following estimate

$$
\varepsilon^{m}\left|\operatorname{vol}\left(V_{\varepsilon, k}^{-}\right)^{-1 / 2} u_{k}-\varepsilon^{-m / 2} u_{j}\left(x^{0}\right)\right|^{2}=\left|\varepsilon^{m / 2}\left(\operatorname{vol} V_{\varepsilon, k}^{-}\right)^{-1 / 2}-\left(\operatorname{vol} V_{k}^{-}\right)^{-1 / 2}\right|^{2}\left|u_{k}\right|^{2}
$$

since $u \in \mathcal{D}_{0}$. The last difference is of order $o(1)$ by (7.8).

7.D. A lower bound. The estimate on $\lambda_{k}\left(M_{\varepsilon}\right)$ from below can be found in analogy with the slowly decaying case in Section 6. Furthermore, we need the following averaging operator

$$
C_{k}^{-} u:=\frac{1}{\operatorname{vol} V_{k}^{-}} \int_{V_{k}^{-}} u d V_{k}^{-} .
$$

Since we have an exact scaling of the metric of order $\varepsilon^{\alpha}$ by (17.6), we also could use the $\varepsilon$-depending manifold $V_{\varepsilon, k}^{-}$here (cf. also Remark [5.7).

Lemma 7.4. For all $u \in \mathcal{H}^{1}\left(V_{\varepsilon, k}^{-}\right)$we have

$$
\left|C_{k}^{-} u-N_{j} u\left(x^{0}\right)\right|^{2} \leq o\left(\varepsilon^{-m}\right)\|d u\|_{V_{\varepsilon, k}}^{2}
$$

Proof. We have

$$
\left|C_{k}^{-} u-N_{j} u\left(x^{0}\right)\right| \leq\left|C_{k}^{-} u-N_{j} u\left(x^{+}\right)\right|+\left|N_{j} u\left(x^{+}\right)-N_{j} u\left(x^{0}\right)\right| .
$$

The first difference can be estimated in the same way as Lemma 5.5 (replacing $V_{k}$ by $V_{k}^{-}$and using estimate (7.7), i.e., we arrive at

$$
\left|C_{k}^{-} u-N_{j} u\left(x^{+}\right)\right|^{2} \leq O\left(\varepsilon^{-(d-2) \alpha}\right)\|d u\|_{V_{\varepsilon}^{-}}^{2} ;
$$

recall that now we have $\alpha d=m$. For the second difference, use (6.17). 
Similarly to Lemma 6.5 we can prove:

Lemma 7.5. For all $u \in \mathcal{H}^{1}\left(V_{\varepsilon}^{-}\right)$, we have

$$
\|u\|_{V_{\varepsilon}^{-}}^{2}-\left\|C^{-} u\right\|_{V_{\varepsilon}^{-}}^{2} \leq O\left(\varepsilon^{\alpha}\right)\left(\|u\|_{V_{\varepsilon}^{-}}^{2}+\|d u\|_{V_{\varepsilon}^{-}}^{2}\right) .
$$

Now we define the transition operator by

$$
\begin{aligned}
\left(\Psi_{\varepsilon} u\right)_{j}(x) & :=\varepsilon^{m / 2}\left(N_{j} u(x)+\rho(x)\left(C_{k}^{-} u-N_{j} u\left(x^{0}\right)\right)\right) \text { for } x \in I_{j k} \\
\left(\Psi_{\varepsilon} u\right)_{k} & :=\varepsilon^{m / 2}\left(\operatorname{vol} V_{k}^{-}\right)^{1 / 2} C_{k}^{-} u
\end{aligned}
$$

where $\rho$ is a smooth function as in (5.11) and $x^{0}=x_{j k}^{0}$ denotes the endpoint of the half-edge $I_{j k}$ corresponding to the vertex $v_{k}$.

Lemma 7.6. We have $\Psi_{\varepsilon} u \in \mathcal{D}_{0}$ if $u \in \mathcal{H}^{1}\left(M_{\varepsilon}\right)$. Furthermore,

$$
\begin{gathered}
\|u\|_{M_{\varepsilon}}^{2}-\left\|\Psi_{\varepsilon} u\right\|_{\mathcal{H}_{0}}^{2} \leq o(1)\left(\|u\|_{M_{\varepsilon}}^{2}+\|d u\|_{M_{\varepsilon}}^{2}\right) \\
q_{0}\left(\Psi_{\varepsilon} u\right)-\|d u\|_{M_{\varepsilon}}^{2} \leq o(1)\left(\|u\|_{M_{\varepsilon}}^{2}+\|d u\|_{M_{\varepsilon}}^{2}\right)
\end{gathered}
$$

for all $u \in \mathcal{H}^{1}\left(M_{\varepsilon}\right)$.

Proof. The arguments are the same as in the proof of Lemma 6.10, For the vertex contribution, we need the estimate

$\|u\|_{V_{\varepsilon, k}^{-}}^{2}-\varepsilon^{m}\left(\operatorname{vol} V_{k}^{-}\right)\left|C_{k}^{-} u\right|^{2}=\left(\|u\|_{V_{\varepsilon, k}^{-}}^{2}-\left\|C_{k}^{-} u\right\|_{V_{\varepsilon, k}^{-}}^{2}\right)+\left(\frac{\operatorname{vol} V_{\varepsilon, k}^{-}}{\varepsilon^{m} \operatorname{vol} V_{k}^{-}}-1\right) \varepsilon^{m}\left\|C_{k}^{-} u\right\|_{V_{k}^{-}}^{2}$.

The first difference can be treated with Lemma [7.5 and leads to an error term $O\left(\varepsilon^{\alpha}\right)$. The second term is of order $o(1)\|u\|_{V_{\varepsilon, k}^{-}}^{2}$ by (7.7), (7.8) and CauchySchwarz. Furthermore, Corollary 6.8 is also true in this setting (independent on the particular $\alpha$ ). We also need Lemma 7.4.

Using Lemma [7.6 we arrive at the sought lower bound. Again, the error term $\eta_{k}$ in (2.11) can be estimated by some $\varepsilon$-independent quantity because $\lambda_{k}=$ $\lambda_{k}\left(M_{\varepsilon}\right) \leq c_{k}$ by Theorem 7.2 .

Theorem 7.7. We have $\lambda_{k}\left(Q_{0}\right) \leq \lambda_{k}\left(M_{\varepsilon}\right)+o(1)$.

Theorem [7.1 now follows easily by combining the last result with Theorem 7.2 ,

\section{NON-DECAYING VERTEX VOLUME}

In this section, we treat the case when the vertex volume does not tend to 0 . In some sense, this case corresponds to $\alpha=0$ in the previous notation but we need more assumptions to precise the convergence of the manifold $V_{\varepsilon, k}$ to a manifold $V_{0, k}$ as $\varepsilon \rightarrow 0$. We cite only the result here since it has already been presented in $\mathrm{P} 03$ or with a more detailed proof in $[\mathrm{P} 00]$. A related result corresponding to the embedded case (see Example 4.1) as in KuZ01 was proven by Jimbo and Morita in JM92 or for manifolds (with non-smooth junctions between edge and vertex neighbourhoods) by Anné and Colbois in AC95. 
Furthermore, we assume that the transversal direction is a sphere, i.e., $F=\mathbb{S}^{m}$. Let $V_{0, k}$ be a compact $d$-dimensional manifold without boundary for $k \in K$. To each edge $j \in J_{k}$ emanating from the vertex $v_{k}$, we associate a point $x_{j k}^{0} \in V_{0, k}$ such that $x_{j k}^{0}\left(j \in J_{k}\right)$ are mutually distinct points with lower bound $2 \varepsilon_{0}>0$ on their distance to each other. We assume for simplicity that the metric at $x^{0}=x_{j k}^{0}$ is locally flat within a distance $\varepsilon_{0}$ from $x^{0}$ (the general case can be found in $[\mathrm{P} 03])$. Then the metric in polar coordinates $(x, y) \in\left(0, \varepsilon_{0}\right) \times \mathbb{S}^{m}$ looks locally like

$$
g=d x^{2}+x^{2} h_{y}
$$

where $h_{y}$ is the standard metric on $\mathbb{S}^{m}$. Modifying the factor before $h_{y}$, we define a new metric by

$$
g_{\varepsilon}=d x^{2}+r_{\varepsilon}^{2}(x) h_{y}
$$

with a smooth monotone function $r_{\varepsilon}:\left(0, \varepsilon_{0}\right) \longrightarrow(0, \infty)$ such that

$$
r_{\varepsilon}(x)= \begin{cases}\varepsilon & \text { for } 0<x<\varepsilon / 2 \\ x & \text { for } 2 \varepsilon<x<\varepsilon_{0} .\end{cases}
$$

We denote the (completion of the) manifold $\left(V_{0, k} \backslash \bigcup_{j \in J_{k}}\left\{x_{j k}^{0} \mid j \in J_{k}\right\}, g_{\varepsilon}\right)$ by $V_{\varepsilon, k}$. Note that this manifold has $\left|J_{k}\right|$ attached cylindrical ends of order $\varepsilon$ at each point $x_{j k}^{0}$. Now we can construct the graph-like manifold $M_{\varepsilon}$ as in Section 3 ,

As in the slowly decaying case of Section [6 the limit operator

$$
Q_{0}:=\bigoplus_{j \in J} \Delta_{I_{j}}^{\mathrm{D}} \oplus \bigoplus_{k \in K} \Delta_{V_{0, k}}
$$

decouples and the next result follows (cf. [P03, Theorem 1.2] or [P00]):

Theorem 8.1. We have $\lambda_{k}\left(M_{\varepsilon}\right) \rightarrow \lambda_{k}\left(Q_{0}\right)$ as $\varepsilon \rightarrow 0$.

\section{Applications}

Finally we comment on consequences of the spectral convergence. We begin with a general remark stating that we only have uniform control over a compact spectral interval:

Remark 9.1. Note that the convergence $\lambda_{k}\left(M_{\varepsilon}\right) \rightarrow \lambda_{k}\left(M_{0}\right)$ cannot be uniform in $k \in \mathbb{N}$ : if this were the case, the theta-function

$$
\Theta_{\varepsilon}(t):=\operatorname{tr} \mathrm{e}^{-t \Delta_{M_{\varepsilon}}}=\sum_{k} \mathrm{e}^{-t \lambda_{k}\left(M_{\varepsilon}\right)}
$$

would converge to $\Theta_{0}(t)$. But Weyl asymptotics are different in the two cases,

$$
\Theta_{\varepsilon}(t) \sim \frac{\operatorname{vol}_{d} M_{\varepsilon}}{(4 \pi t)^{d / 2}}, \quad \text { whereas } \quad \Theta_{0}(t) \sim \frac{\operatorname{vol}_{1} M_{0}}{(4 \pi t)^{1 / 2}}
$$

as $t \rightarrow 0$ (cf. [Ch84, Sec. VI.4] and [Ro84, Thm. 1]). Recall that $d \geq 2$ and $\operatorname{vol}_{1} M_{0}:=\sum_{j} \ell_{j}$, i.e. the sum over the length of each edge. 
9.A. Periodic graphs. Suppose we have an infinite graph $X_{0}$ on which a discrete, finitely generated group $\Gamma$ operates such that the quotient $M_{0}:=X_{0} / \Gamma$ is a finite graph. In the same way as in the previous sections, we can associate a family of graph-like compact manifolds $M_{\varepsilon}$ to the graph $M_{0}$. By a lifting procedure we obtain a (non-compact) covering manifold $X_{\varepsilon}$ of $M_{\varepsilon}$ with deck transformation group $\Gamma$, i.e., $M_{\varepsilon}$ is isometric to $X_{\varepsilon} / \Gamma$. Furthermore, $X_{\varepsilon}$ is a graph-like manifold collapsing to the infinite graph $X_{0}$.

We are interested in spectral properties of the non-compact manifolds $X_{\varepsilon}$. Assuming that $\Gamma$ is abelian, we can apply Floquet theory (for a non-commutative version see [LP04]). Instead of investigating $\Delta_{X_{\varepsilon}}$ we analyze a family of operators $\Delta_{M_{\varepsilon}}^{\theta}, \theta \in \hat{\Gamma}$, where $\hat{\Gamma}$ is the dual group, i.e., the group of homomorphisms from $\Gamma$ into the unit circle $\mathbb{T}^{1}$. The operator $\Delta_{M_{\varepsilon}}^{\theta}$ acts on a complex line bundle over the compact manifold $M_{\varepsilon}$, or equivalently, over the closure of a fundamental domain $D_{\varepsilon} \subset X_{\varepsilon}$ with $\theta$-periodic boundary conditions. We call the closure $\bar{D}_{\varepsilon}$ a period cell and denote it also by $M_{\varepsilon}$ (for details see e.g. [RS-4] or [P03]). The direct integral decomposition implies

$$
\operatorname{spec} \Delta_{X_{\varepsilon}}=\bigcup_{k \in \mathbb{N}} B_{k}(\varepsilon), \quad B_{k}(\varepsilon):=\left\{\lambda_{k}^{\theta}\left(M_{\varepsilon}\right) \mid \theta \in \hat{\Gamma}\right\}
$$

where $B_{k}(\varepsilon)$ is a compact subset of $[0, \infty)$, called the $k$-th band. ${ }^{6}$ A similar assertion holds for the limit operator on $X_{\varepsilon}$.

9.B. Spectral gaps. We are interested in the existence of spectral gaps of the operator $\Delta_{X_{\varepsilon}}$, i.e., the existence of an interval $[a, b], 0<a<b$, such that $\operatorname{spec} \Delta_{X_{0}} \cap[a, b]=\emptyset$. Note that spec $\Delta_{X_{\varepsilon}}$ is purely essential.

Theorem 9.2. We have $\lambda_{k}^{\theta}\left(M_{\varepsilon}\right) \rightarrow \lambda_{k}^{\theta}\left(Q_{0}\right)$ for $\varepsilon \rightarrow 0$ uniformly in $\theta \in \hat{\Gamma}$. Furthermore,

$$
B_{k}(\varepsilon) \cap B_{k+1}(\varepsilon)=\emptyset \quad \text { if } \quad B_{k}(0) \cap B_{k+1}(0)=\emptyset
$$

provided $\varepsilon$ is small enough. In particular, an arbitrary (but finite) number of gaps open up in the spectrum of $\Delta_{X_{\varepsilon}}$ provided the limit operator $Q_{0}$ has enough gaps and $\varepsilon$ is small enough.

Proof. The spectral convergence can be proven in the same way as in the previous sections. Note that the error terms converge uniformly in $\theta \in \hat{\Gamma}$ since all error bounds are independent of $\theta$. The only point where $\theta$ enters is the error estimate (2.11) for the lower eigenvalue estimate. In this case, we argue as follows: we have $\lambda_{k}^{\theta}\left(M_{\varepsilon}\right) \leq \lambda_{k}^{\mathrm{D}}\left(M_{\varepsilon}\right)$, i.e., the Dirichlet Laplacian eigenvalues form an upper bound on the $\theta$-periodic eigenvalues. Here, we pose Dirichlet boundary conditions on the boundary of the period cell. Furthermore, $\lambda_{k}^{\mathrm{D}}\left(M_{\varepsilon}\right) \rightarrow \lambda_{k}^{\mathrm{D}}\left(M_{0}\right)$

\footnotetext{
${ }^{6}$ Note that $\hat{\Gamma}$ is connected iff $\Gamma$ is torsion free, e.g., if $\Gamma=\mathbb{Z} \times \mathbb{Z}_{2}$ then $\hat{\Gamma} \cong \mathbb{T}^{1} \times \mathbb{Z}_{2}$ which is homeomorphic to two disjoint copies of the unit circle $\mathbb{T}^{1}$. Therefore, the bands $B_{k}(\varepsilon)$ being the continuous image of $\hat{\Gamma}$ under the map $\theta \mapsto \lambda_{k}^{\theta}\left(M_{\varepsilon}\right)$ need not to be intervals.
} 
by the same arguments as in the previous sections. Therefore, we can choose $\lambda_{k}=\lambda_{k}^{\theta}\left(M_{\varepsilon}\right) \leq \lambda_{k}^{\mathrm{D}}\left(M_{\varepsilon}\right) \leq 2 \lambda_{k}^{\mathrm{D}}\left(M_{0}\right)$ in (2.11) independently of $\theta$.

Note that we cannot expect to show the existence of infinitely many gaps in $X_{\varepsilon}$ even if spec $\Delta_{X_{0}}$ has infinitely many gaps since the convergence is not uniform in $k$ (cf. Remark 9.1). This is related to the deep open problem about the validity of Bohr-Sommerfeld conjecture on such periodic manifolds.

Remark 9.3. If two neighboured bands $B_{k}(0)$ and $B_{k+1}(0)$ overlap, i.e., intersect in a set of positive length, the same is true for $B_{k}(\varepsilon)$ and $B_{k+1}(\varepsilon)$ provided $\varepsilon$ is small enough. In contrast, if the bands intersect only in one point, i.e., if they touch each other, we cannot say anything about the (non-)existence of gaps in the spectrum of $\Delta_{X_{\varepsilon}}$.

For the rest of this section we discuss examples for which Theorem 9.2 applies.

9.C. Decoupling limit operators. Suppose that our graph-like periodic manifold $X_{\varepsilon}$ is constructed as in Section [6] or 8. In this case, the limit operator is a direct sum of the limit operator on the quotient $M_{0}$ since the limit operator decouples. Therefore, the bands $B_{k}(0)$ degenerate to the points $\lambda_{k}\left(Q_{0}\right)$ where $Q_{0}$ is given as in Sections 6 or 8 and the limit operator on $X_{0}$ has infinitely many gaps. This means, in particular, that the limit spectrum is not absolutely continuous, while those of the approximating operators may be. Furthermore, Theorem 9.2 applies in this case.

9.D. Cayley graphs and Kirchhoff boundary conditions. In the following three subsections, we give examples of graph-like manifolds with fast decaying vertex volume as constructed in Section 5 such that $\Delta_{X_{\varepsilon}}$ has spectral gaps. In this case, the limit operator is the Laplacian $\Delta_{X_{0}}$ on the graph $X_{0}$ with Kirchhoff boundary conditions as in (2.3). We want to calculate the spectrum of $\Delta_{X_{0}}$ for certain graphs $X_{0}$. For simplicity, we assume that $p_{j} \equiv 1$ and that each edge has length 1.

Suppose that $\Gamma$ is an abelian, finitely generated discrete group. Therefore,

$$
\Gamma \cong \mathbb{Z}^{r_{0}} \times \mathbb{Z}_{p_{1}}^{r_{1}} \times \cdots \times \mathbb{Z}_{p_{a}}^{r_{a}}
$$

where $\mathbb{Z}_{p}$ is the cyclic group of order $p$. Furthermore, $r_{0}>0$ since $X_{0}$ is noncompact and $X_{0} / \Gamma$ is compact. Denote $r:=r_{0}+r_{1}+\cdots+r_{a}$.

We assume that $X_{0}$ is the (metric) Cayley graph associated to $\Gamma$ w.r.t. the canonical generators $\varepsilon_{1}, \ldots, \varepsilon_{r}$ ( $\varepsilon_{j}$ equals 1 at the $j$-th component and 0 otherwise), i.e., the set of vertices is $\Gamma$ and two vertices $\gamma_{1}, \gamma_{2}$ are connected iff $\gamma_{2}=\varepsilon_{j} \gamma_{1}$ for some $1 \leq j \leq r$ (see Figure 5). Note that $X_{0}$ is $2 r$-regular, i.e., each vertex meets $2 r$ edges. We want to calculate the eigenfunctions and eigenvalues of the $\theta$-periodic operator $\Delta_{M_{0}}^{\theta}$, i.e., functions $u_{j}$ on $I_{j} \cong[0,1]$ satisfying $-u_{j}^{\prime \prime}=\lambda u_{j}$ 

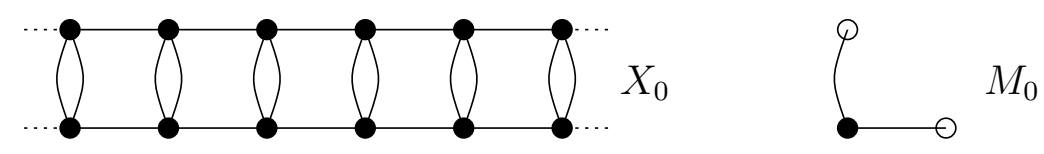

Figure 5. The Cayley graph associated to the group $\Gamma=\mathbb{Z} \times \mathbb{Z}_{2}$ and the corresponding period cell. Note that $\Delta_{X_{0}}$ has no spectral gaps

with the boundary conditions

$$
u_{j}(0)=u(0), \quad \mathrm{e}^{-\mathrm{i} \theta_{j}} u_{j}(1)=u(0) \quad \text { and } \quad \sum_{k=1}^{r}\left(\mathrm{e}^{-\mathrm{i} \theta_{k}} u_{k}^{\prime}(1)-u_{k}^{\prime}(0)\right)=0
$$

for all $j=1, \ldots, r$. Here, $\theta \in \mathbb{T}^{r_{0}} \times \mathbb{T}_{p_{1}}^{r_{1}} \times \cdots \times \mathbb{T}_{p_{a}}^{r_{a}}$ where $\mathbb{T}_{p}:=\left\{\xi \in \mathbb{R} / \mathbb{Z} \mid \mathrm{e}^{\mathrm{i} \xi p}=\right.$ $1\}$ is the group of $p$-th unit roots (isomorphic to $\mathbb{Z}_{p}$ ). Note that we have identified $\theta \in \mathbb{T}^{r}$ with $\gamma \mapsto \mathrm{e}^{\mathrm{i} \theta \cdot \gamma} \in \hat{\Gamma}$.

If $\lambda=\omega^{2}>0$ (and $\omega>0$ ) we make the Ansatz

$$
u_{j}(x):=Z \cos (\omega x)+A_{j} \sin (\omega x) .
$$

Non-trivial solutions of the eigenvalue problem exist iff $\omega=\ell \pi, \ell \in \mathbb{N}$, or

$$
\cos \omega=\frac{1}{r} \sum_{k=1}^{r} \cos \theta_{k}
$$

The solutions $\omega=\ell \pi$ correspond to Dirichlet eigenfunctions on each edge and produce therefore bands degenerated to a point $\left\{(\ell \pi)^{2}\right\}$. The multiplicity is $r-1$ provided $\theta \neq 0$ (if $\ell$ is even) resp. $\theta \neq \pi$ (if $\ell$ is odd) and $r+1$ if $\theta=0$ resp. $\theta=\pi$ (modulo $2 \pi$ ). If $\omega \neq \ell \pi$, the eigenvalues are simple. Note that the bands at $\omega^{2}=(\ell \pi)^{2}$ do not overlap, but touch each other.

For $\omega=0$, we need a special Ansatz. The only possibility is the case of periodic boundary conditions $(\theta=0)$; the eigenvalue is simple.

Theorem 9.4. If one of the orders $p_{1}, \ldots, p_{a}$ is odd, the operator $\Delta_{X_{0}}$ has infinitely many spectral gaps below and above $(2 \ell+1)^{2} \pi^{2}(\ell=0,1, \ldots)$. In particular, Theorem 9.2 applies. Furthermore, the bands $\left\{(2 \ell+1)^{2} \pi^{2}\right\}$ are degenerated to a point and have multiplicity $r-1$. The gap length increases as $\ell \rightarrow \infty$.

If all orders $p_{1}, \ldots, p_{a}$ are even then $\operatorname{spec} \Delta_{X_{0}}=[0, \infty)$.

Proof. We analyze the behaviour of $\omega$ in dependence of the continuous parameters $\theta_{1}, \ldots, \theta_{r_{0}} \in \mathbb{T}^{r_{0}}$ given by the relation (9.3). We have gaps iff $\frac{1}{r} \sum_{k=1}^{r} \cos \theta_{k}$ in (9.3) does not cover the whole interval $[-1,1]$. We reach the maximal value 1 iff all $\theta_{j}=0(j=1, \ldots, r)$ and the minimal value -1 iff all $\theta_{j}=\pi(j=1, \ldots, r)$. The latter can only occur if all group orders are even. Note that in this case, the whole interval $[-1,1]$ can be covered by an appropriate choice of the $\theta_{j}$ 's, 
$j=r_{0}+1, \ldots, r$. If one $p_{j}$ is odd, there exists $\varepsilon>0$ such that (9.3) has no solution provided $(2 \ell+1) \pi-\varepsilon<\omega<(2 \ell+1) \pi+\varepsilon$.

We cannot say anything about the (non-)existence of gaps in the case when all orders $p_{1}, \ldots, p_{a}$ are even. If e.g. $\Gamma=\mathbb{Z} \times \mathbb{Z}_{2}$, the bands do not overlap, but touch each other and fill the whole half line $[0, \infty)$ (cf. Remark 9.3).

9.E. Non-commutative groups. We comment briefly on a similar result for certain non-commutative groups $\Gamma$. Here, $\hat{\Gamma}$ consists of (equivalence classes of) irreducible unitary representations (cf. [LP04]). A simple example is given by $\Gamma=\mathbb{Z} \times D_{n}$, where $D_{n}$ denotes the dihedral group of order $2 n$ generated by $\alpha, \beta$ with $\alpha^{2}=e, \beta^{n}=e$ and $\alpha \beta=\beta^{-1} \alpha$. In this case the Laplacian on the
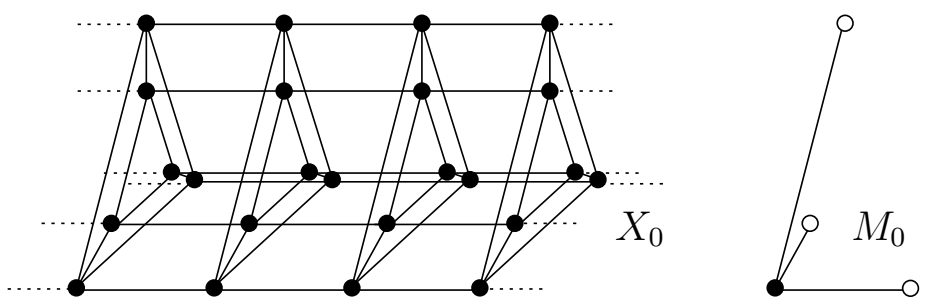

Figure 6. The Cayley graph associated to the group $\Gamma=\mathbb{Z} \times D_{3}$ where $D_{3}$ is the dihedral group of order 6 . The corresponding Laplacian has spectral gaps.

corresponding Cayley graph (cf. Figure 6) has infinitely many spectral gaps below and above $(2 \ell+1)^{2} \pi^{2}, \ell=0,1, \ldots$, if $n$ is odd. If $n$ is even, spec $\Delta_{X_{0}}=[0, \infty)$. For a related family of sleeve manifolds in case of odd $n$ there is an arbitrary large number of open gaps provided the sleeve radius is small enough.

9.F. Cayley graphs with loops. If we set one (or more) of the group orders $p_{j}$ equal to 1 we formally attach a loop (or more) at each vertex (see Figure 7).
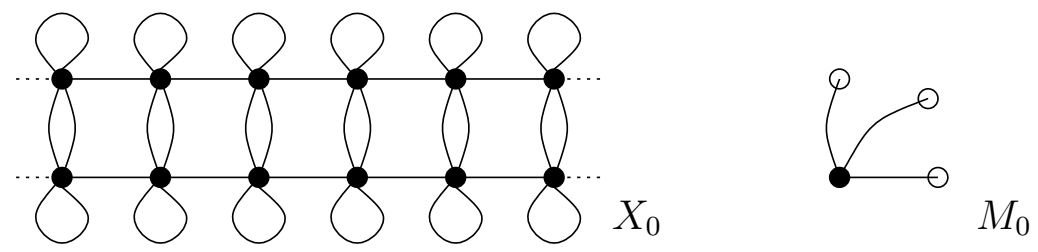

FigurE 7 . The Cayley graph associated to the group $\Gamma=\mathbb{Z} \times \mathbb{Z}_{2} \times$ $\mathbb{Z}_{1}$, where the trivial group $\mathbb{Z}_{1}$ leads to the attachment of a loop at each vertex. On the right, the corresponding period cell is shown. Note that $\Delta_{X_{0}}$ has spectral gaps in contrast to the example without loops. 
Theorem 9.5. The Laplacian of a Cayley graph associated to an arbitrary finitely generated abelian discrete group $\Gamma$ has infinitely many spectral gaps provided we attach at each vertex a fixed number of loops.

Proof. Formally, the assertion follows from Theorem 9.4. Note that $\hat{\mathbb{Z}}_{1}=\{0\}$, i.e., the corresponding component of $\theta$ cannot be $\pi$ and therefore, the minimum -1 cannot be achieved in (9.3).

This is an analogue of gap generation by decoration of the graph as discussed by Aizenman and Schenker in AS00].

9.G. Cayley graphs and the borderline case. In the borderline case, the eigenvalue problem of the limit operator is more complicated. Here, functions $u_{j}$ on $I_{j} \cong[0,1]$ satisfy $-u_{j}^{\prime \prime}=\lambda u_{j}$ with the boundary conditions

$$
u_{j}(0)=u(0), \quad \mathrm{e}^{-\mathrm{i} \theta_{j}} u_{j}(1)=u(0) \quad \text { and } \quad \sum_{k=1}^{r}\left(\mathrm{e}^{-\mathrm{i} \theta_{k}} u_{k}^{\prime}(1)-u_{k}^{\prime}(0)\right)=c \lambda u(0)
$$

for all $j=1, \ldots, r$ where $c^{2}$ is the volume of the (unscaled) vertex neighbourhood (cf. (7.3)). Again, with the Ansatz (9.2), non-trivial solutions of the eigenvalue problem exist iff $\omega=\ell \pi, \ell \in \mathbb{N}$, or

$$
\cos \omega-\frac{\omega \sin \omega}{2 r c}=\frac{1}{r} \sum_{k=1}^{r} \cos \theta_{k} .
$$

Note that formally the case $c=0$ corresponds to the Kirchhoff boundary condition case. Again, the solutions $\omega=\ell \pi$ belong to Dirichlet eigenfunctions on each edge and produce therefore bands degenerated to a point $\left\{(\ell \pi)^{2}\right\}$.

Theorem 9.6. The limit operator $Q_{0}$ in the borderline case defined on a Cayley graph associated to an arbitrary finitely generated abelian discrete group $\Gamma$ has infinitely many spectral gaps located around $(2 \ell+1)^{2} \pi^{2} / 4$ provided $\ell$ is large enough. If at least one group order $p_{j}$ is odd, we have also spectral gaps below and above $(2 \ell+1)^{2} \pi^{2}$ for $\ell=0,1, \ldots$

Proof. The function $f(\omega):=\cos \omega-\omega \sin \omega /(2 r c)$ oscillates with an amplitude of order $\omega$. In particular, for $\omega=(2 \ell+1) \pi / 2$ we have $|f(\omega)|=(2 \ell+1) \pi /(4 r c)$, i.e., there is no solution of (9.5) provided $\ell$ is large enough. Furthermore, since $f((2 \ell+1) \pi)=-1$, we can argue as in Theorem 9.4 .

Returning to our graph-like periodic manifolds, we have the following situation: In the case of fast decaying vertex volume, i.e. $(d-1) / d<\alpha \leq 1$, we have spectral gaps below and above $(2 \ell+1)^{2} \pi^{2}$ provided at least one order $p_{j}$ is odd. In particular, $(2 \ell+1)^{2} \pi^{2}$ is an isolated eigenvalue (degenerated band). In the borderline case, these gaps remain open. Furthermore, we always have spectral gaps around $(2 \ell+1)^{2} \pi^{2} / 4$ provided $\ell$ is large enough. In the case of slowly 
decaying vertex volume, i.e., $0<\alpha<(d-1) / d$, all bands concentrate around $\ell^{2} \pi^{2}$, i.e. we have large gaps around $(2 \ell+1)^{2} \pi^{2} / 4$ for all $\ell=0,1, \ldots$

The situation is more complicated if we allow different length ratios for the edges. In such a case the spectrum could be more complicated; recall the example of a lattice graph discussed in [EG96] shows where number-theoretic properties of parameters play a rôle. This interesting question will be considered separately.

\section{ACKNOWLEDGEMENTS}

The authors appreciate P. Kuchment who made available to them the paper KuZ03 prior to publication. O.P. is grateful for the hospitality extended to him at the Nuclear Physics Institute of Czech Academy of Sciences where a part of this work was done. The research was partially supported by the GAAS grant A1048101 and the SPECT program of the European Science Foundation.

\section{REFERENCES}

[AGHH04] S. Albeverio, F. Gesztesy, R. Høegh-Krohn, H. Holden: Solvable Models in Quantum Mechanics, appendix to the 2nd edition, Chelsea Publ. Co. 2004; to appear

[AC95] C. Anné and B. Colbois, Spectre du Laplacien agissant sur les p-formes différentielles et écrasement d'anses, Math. Ann. 303 (1995), no. 3, 545-573.

[AMSC01] A. N. Andriotis, M. Menon, D. Srivastava, and L. Chernozatonskii, Rectification properties of carbon nanotube "Y-junctions", Phys. Rev. Lett. 87 (2001), 066802.

[AS00] M. Aizenman and J. H. Schenker, The creation of spectral gaps by graph decoration, Lett. Math. Phys. 53 (2000), 253-262.

[CdV86] Y. Colin de Verdière, Sur la multiplicité de la première valeur propre non nulle du laplacien, Comment. Math. Helv. 61 (1986), no. 2, 254-270.

[Ch84] I. Chavel, Eigenvalues in Riemannian geometry, Academic Press, Orlando, 1984.

[Da96] E. B. Davies, Spectral theory and differential operators, Cambridge University Press, Cambridge, 1996.

[DE95] P. Duclos, P. Exner, Curvature-induced bound states in quantum waveguides in two and three dimensions, Rev. Math. Phys. 7 (1995), 73-102.

[EG96] P. Exner and R. Gawlista, Band spectra of rectangular graph superlattices, Phys. Rev. B 53 (1996), no. 11, 7275-7286.

[EŠ89] P. Exner and P. Šeba, Bound states in curved quantum waveguides, J. Math. Phys. 30 (1989), no. 11, 2574-2580.

[JM92] Sh. Jimbo and Y. Morita, Remarks on the behavior of certain eigenvalues on a singularly perturbed domain with several thin channels, Commun. Partial Differ. Equations 17 (1992), 523-552.

[K66] T. Kato, Perturbation theory for linear operators, Springer-Verlag, Berlin, 1966.

[KoS99] V. Kostrykin and R. Schrader, Kirchhoff's rule for quantum wires, J. Phys. A 32 (1999), no. 4, 595-630.

[Ku02] P. Kuchment, Graph models for waves in thin structures, Waves Random Media 12 (2002), R1-R24.

[Ku04] P. Kuchment, Quantum graphs: I. Some basic structures, Waves in Random Media 14 (2004), S107-128.

[KuZ01] P. Kuchment and H. Zeng, Convergence of spectra of mesoscopic systems collapsing onto a graph, J. Math. Anal. Appl. 258 (2001), no. 2, 671-700. 
[KuZ03] _ Asymptotics of spectra of Neumann Laplacians in thin domains, Advances in differential equations and mathematical physics (Birmingham, AL, 2002), Contemp. Math., vol. 327, Amer. Math. Soc., Providence, RI, 2003, pp. 199-213.

[LP04] F. Lledó and O. Post, Generating spectral gaps by geometry, Preprint (2004).

[P00] O. Post, Periodic manifolds, spectral gaps, and eigenvalues in gaps, Ph.D. thesis, Technische Universität Braunschweig, 2000.

[P03] - Periodic manifolds with spectral gaps, J. Diff. Equations 187 (2003), 23-45.

[PRL00] C. Papadopoulos, A. Rakitin, J. Li, V. S. Vedeneev, and J. M. Xu, Electronic properties in $Y$-junction carbon nanotubes, Phys. Rev. Lett. 85 (2000), 3476-3479.

[Ro84] J.-P. Roth, Le spectre du laplacien sur un graphe, Théorie du potentiel (Orsay, 1983), Lecture Notes in Math., vol. 1096, Springer, Berlin, 1984, pp. 521-539.

[RueS53] K. Ruedenberg and C. W. Scherr, Free-electron network model for conjugated systems, I. Theory, J. Chem. Phys. 21 (1953), 1565-1581.

[RS-1] M. Reed and B. Simon, Methods of modern mathematical physics I: Functional analysis, Academic Press, New York, 1980.

[RS-4] L Methods of modern mathematical physics IV: Analysis of operators, Academic Press, New York, 1978.

[RubS01] J. Rubinstein and M. Schatzman, Variational problems on multiply connected thin strips. I. Basic estimates and convergence of the Laplacian spectrum, Arch. Ration. Mech. Anal. 160 (2001), no. 4, 271-308.

[Sa00] Y Saito, The limiting equation for Neumann Laplacians on shrinking domains., Electron. J. Differ. Equ. 31 (2000), 25 p.

[Tay96] M. E. Taylor, Partial differential equations. Basic theory, Springer-Verlag, New York, 1996.

[TBG02] M. Terrones, F. Banhart, N. Grobert, J.-C. Charlier, H. Terrones, and P. M. Ajayan, Molecular junctions by joining single-walled carbon nanotubes, Phys. Rev. Lett. 89 (2002), 075505.

Department of Theoretical Physics, NPI, Academy of Sciences, 25068 Řež near Prague, and Doppler Institute, Czech Technical University, Břehová 7, 11519 Prague, Czechia

Institut für Reine und Angewandte Mathematik, Rheinisch-Westfälische Technische Hochschule Aachen, Templergraben 55, 52062 Aachen, Germany 